\title{
ON THE RENORMALIZATION METHOD:
}

\section{A DIFFERENT OUTLOOK, AND THE ENERGY-MOMENTUM}

TENSOR IN THE $9 \phi^{4}$ THEORY

\author{
Enrico C. Poggio \\ Department of Physits \\ Columbia University, New York; N. Y.
}

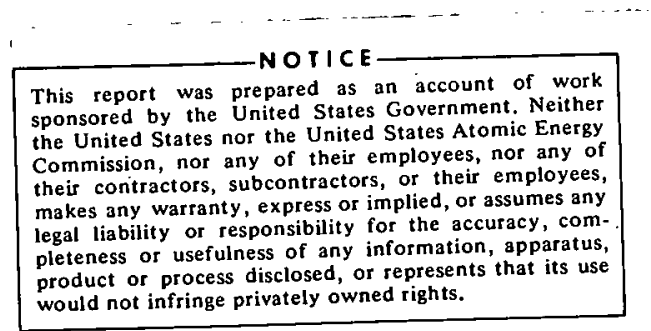

This research was supported in part by the U. S. Atomic Energy Commi ision. 


\section{DISCLAIMER}

This report was prepared as an account of work sponsored by an agency of the United States Government. Neither the United States Government nor any agency Thereof, nor any of their employees, makes any warranty, express or implied, or assumes any legal liability or responsibility for the accuracy, completeness, or usefulness of any information, apparatus, product, or process disclosed, or represents that its use would not infringe privately owned rights. Reference herein to any specific commercial product, process, or service by trade name, trademark, manufacturer, or otherwise does not necessarily constitute or imply its endorsement, recommendation, or favoring by the United States Government or any agency thereof. The views and opinions of authors expressed herein do not necessarily state or reflect those of the United States Government or any agency thereof. 


\section{DISCLAIMER}

Portions of this document may be illegible in electronic image products. Images are produced from the best available original document. 


\begin{abstract}
A new method of renormalizing a quartic self-interacting boson theory has been developed. The need to introduce a Lagrangian with counterterms and renormalization constants is avoided and a simple and compact set of computational rules results. As a consequence of the propagator function analysis, one is able to renormalize explicitly the field energy-momentum tensor to all orders. One finds a one-parameter family of renormalizable tensors, none of which is the one proposed by Callan, Coleman and Jackiw. The method will allow for a simple determination of the asymptotic "zeromass" theory.
\end{abstract}




\section{INTRODUCTION}

It is a well known fact that the scalar field theory with quartic self-interaction is of the renormalizable type. A decade ago, $W_{u}(1)$, in an attempt to formulate a dynamical field theoretic description of interacting pions, made a complete, extensive and complicated study of the renormalization program for the above theory by means of a well known, standard method $(2),(\beta)$. Between then and the present time, various alternatives $(4),(5),(6)$, some of them resulting as an outgrowth of the problems of broken scale invoriance and the short distance behovior of field theories $(7),(8),(9)$, have been widely discussed in the literature.

It is our opinion, nevertheless, that such a seemingly well known and understood problem should deserve to be studied, profitably, once more from a different point of view. As it will be argued, the profits to be gained are two-fold. Firstly, we believe, we will introduce a great deal of formal simplification to the whole idea behind renormalization programs. This simplification is also readily reflected, as a concrete end product, by a compact and clear calculational scheme. Secondly, as a direct consequence of our renormalization philosophy, one will have at hand a formalism from which one can also easily study the inherent short-distance behovior of the theory $(10)$.

The standard, "canonical" renormalization formalism consists in recognizing the fact that the "bare" field Lagrangian yields divergent results when one computes the relevant Green's functions. The nature of such divergences can be understood in terms of the "bare" masses and coupling constants being divergent, undefined quantities. One then rewrites the Lagrangian in terms of finite, well-defined, physical, masses 
and couplings, by properly adding divergence-cancelling counterterms, so that the theory, when expressed in terms of these new parameters, will be finite (at least in perturbation theory). The "bare" parameters and the physical parameter are connected by the infinite renormalization constants which appropriately make up the Lagrangian counterterms. The success of the program depends upon properly defining such constants.

We will take the attitude that this whole procedure is too formal, cumbersome and unnecessary, and that one can obtain the same finite, renormalized results in a much more clear and explicit way if the problem is analyzed from a proper perspective. The existence of a Lagrangian or the nature of the renormalization constants will bear no direct consequences on our results. Our renormalization procedure consists of, essentially, three steps. First, we will try to obtain a formal "Dyson-Schwinger" type equation obeyed by the Green's functions. By formal, we shall mean that, although they may be of little quantitative value (for they may be divergent), they contain all the typical structures and characteristics required by the nature of the interaction; their graphical solution should generate all the appropriate Feynman diagrams with the relevant invariance and symmetry properties. The second step will consist in "reducing" the above formal equations into finite, explicit ones. Such a "reduction" is carried out by studying and understanding the divergent structure of the formal equations and then by applying, accordingly, a well defined sequence of differential operations which will annihilate all the divergent parts. The reduced, finite set will then represent integral-differential forms of the Green's functions. In certain cases, for convenience, and in order to make certain formal manipulations in the above operations valid, it is convenient to introduce a large, but finite, external cut-off 
into the theory. As long as the cut-off is finite, the formal, but now cut-off dependent, equations will be finite. By letting the cut-off go to infinity, the original divergence structure will be recuperated. When the proper choice of the differential annihilators is made, the infinite cut-off limit will give a unique, well-defined result. The introduction of the cut-off can be made by adding, for example, a term $q / \Lambda^{2}$, $\wedge$ being the cut-off, to the propagator function $\Delta^{-1}\left(q^{2}\right)$, which for mnemonical reasons only, can be thought of as coming from the addition of a term $\frac{\left(D^{2} \varphi\right)^{2}}{\Lambda^{2}}$ to the kinetic energy of the bare Lagrangian (11), (12). The program will be completed. upon specifying a set of boundary conditions which allow for a unique integration of the differentiated Green's functions obtained from the reduction of the formal equations. It is through these conditions that the physical parameters will be introduced into the theory. Let us remark that this program doesn't represent anything that is radically new. Many of the ideas have been, are, and probably will be again expressed in some form or another. Our goal, through a re-orientation of ideas, is only to bring a new, and we think better, perspective to such an old and well thought-out problem.

The necessity of divorcing ourselves from canonical renormalization procedures became evident while studying the short-distance behavior of renormalizable field theories, in the sense of Gell-Mann and Low $(10),(13)$. It was then realized that such renormalization procedures were not able to produce simply and explicitly the limiting "zero-mass" theory which is critical, in the Gell-Mann and Low theory, for the derivation of what are now known as the renormalization group equations ${ }^{(14)}$, together with their interesting consequences and conjectures $(10),(15),(16)$. Not surprisingly, the stumbling block comes from the renormalization constants which act as connector 
functions between the "zero-mass" functions and parameters and the corresponding renormalized ones. On the other hand, our choice of renomalization procedure seems to give, naturally, a smooth limit to the "zero-mass" region, the connecting functions being a well-defined combination of known functions. It is in this sense that our renormalization procedure plays a crucial role in the development of the larger program studying the asymptotic behavior of field theories.

The apparent "non-commutativity" between canonical renormalization and short-distance asymptotia is, "seemingly, a more general aspect of relativistic field theories $(17)$. Contrary to the non-relativistic case, at short distances, the kinetic energy part and the interaction part of a Lagrangian density cannot be distinguished, in general. Effectively, this means that there is no internal scale that could separate out the various kinematical and dynamical regions. At short distances, the commutation relations will become non-canonical, interaction-dependent. This fact is reflected by the behavior of the commutator function. When $q^{2} \gg m^{2}$ ( $m$ is the physical mass)

$$
\lim _{q^{2} \gg m^{2}} q^{2} \Delta\left(q^{2}\right)=\lim _{q^{2} \rightarrow \infty} F\left(\frac{q^{2}}{m^{2}} j q\right)
$$

The function $F$ is undetermined by the canonical formalism. In perturbation theory, $F$ is seen to be, in general, a divergent function. On the other hand, as $q^{2} \rightarrow-m^{2}$

$$
\lim _{q^{2} \rightarrow-m^{2}}\left(q^{2}+m^{2}\right) \Delta\left(q^{2}\right)=Z
$$

where $Z$ is one of the renormalization constants. The non-uniformity of behavior is quite evident. The definition of the renormalized constants always comes from a region 
of space away from the short-distance region, and its connection to it is not explicit.

In this paper, we will carry out the proposed renormalization program for the $\varphi^{4}$ theory vertex and propagator function. Interestingly enough, because of the constraints of our program, the study of the propagator function equations is seen to be effectively carried out by introducing zero-energy graviton insertions. The tensor vertex thus defined can be related to the matrix elements of the relevant Energy-Momemtum tensor in the forward direction. The analysis can then be easily extended to the non-forward case, and explicit, well-defined renormalization equations for the E-M tensor are abtained. Some time ago Callan, Coleman and Jackiw ${ }^{(18)}$ argued for the existence of a special tensor which would have the properties that it could be used as the generator of (broken) scale and conformal transformations and that its matrix elements would be finite. The conclusions drawn from our equations indicate that there is no such preferred tensor. Even though the CCJ tensor is, formally, the indicated one to study such symmetry breaking, perturbation theory tells us that there is no renormalizable tensor with such properties. Surprisingly we also find the existence of a one-parameter family of renomalizable tensors $(7)$. The parameters are arbitrary and independent of any requirements imposed by either perturbation theory or broken scale symmetry.

The matter of the short-distance behavior will be taken up in a separate paper (19) The "zero-mass" equations will be derived. These will yield, exactly, the renormalization group equations, which will be studied in detail when the interesting, but yet. physically unclear, eigenvalue condition is assumed. Furthermore we will make there explicit calculational use of the formalism presented in the present paper. The "zeromass" function equations are of the exact same form as the renormalized, "massive" 
equations, but they have the added advantage of depending on simpler structure propagator functions which allow straightforward evaluation of relevant integrals. In a sense the "zero-mass" theory is an application of our renormalization formalism.

This study is divided into four chapters. The first will just spell out the basic language and formalism to be used. The mechanism of the program will be re-expressed in more specific terms relevant to the theory in question. Assuming that the propagator is known, the vertex equations are obtained in the second chapter. The third chapter. contains the analysis of the propagator function. It is shown there that the invariance of the interaction under general linear coordinate transformations allows us to study the problem in terms of zero-groviton insertions and the corresponding energy-momentum tensor vertex and Ward Identities. The generalization to the non-forward case is done in the last chapter. 
Chapter I

Renormalization Preliminaries

Keeping in mind our stated renormalization "philosophy", we will next proceed to carry out in some detail our program for the Green's functions of the theory that are of interest to us, namely the two-point function (propagator) and the fourpoint function (vertex). Our purpose is to obtain a set of consistent, explicit and simple equations for these functions which could then be solved explicitly by iteration to yield the well known perturbation expansions to all orders in the physical coupling.

For the sake of completeness, and also partly to motivate the construction of our theory, we will briefly spell out the classification of concepts which are relevant in carrying out any renormalization procedure, in general. More formal and complete discussions can be found in the extensive literature on the subject $(1),(6)$.

First, let us introduce the concept of One Particle Irreducible (O.P. I.) contributions to the propagator and vertex functions, also known as the proper parts. An O.P. I. graph is one which cannot be broken up by cutting a single internal line (Fig. 2). These are the only ones that need to be studied (all vertices are O.P. I. in this theory), for the remaining contributions the One Particle Reducible (O.P. R.) or improper graphs are just combinations of O.P. I. connected by single internal lines. Next, in order to study the nature of the divergences of the theory, we introduce the concept of primitive or skeleton graphs. These belong to a subclass of O.P.I. graphs which are obtained by shrinking the internal propagators and vertices of a given O.P. I. graph into lines and points respectively (Fig. 3). Well known counting arguments indicate that the characteristic superficial divergence of a given graph is 
determined only by the number of exterior lines. For a boson theory, this is given by

$$
D=4-N_{b}
$$

where $D$ is the degree of divergence, and $N_{b}$ is the number of external boson lines. The characteristic divergences of the primitive graphs will be the same as the overall divergences of the corresponding non-primitive ones. From (1. 1) the primitive divergence of the vertex and propagator will be logarithmic and quadratic respectively. All higher point functions are convergent. In some sense the primitive divergences characterize a given renormalizable theory, for they give us an indication of how many "subtractions" one has to make in order to make the theory finite. In this case, we will need two for the propagator and one for the vertex. From a more analytical point of view this reduction from infinite to finite Green's function means that we must have a second order differential equation for the propagator and a first order one for the vertex. By subtractions we then mean that we have to specify enough boundary conditions to solve the equations uniquely. In our case we must specify, at some convenient point, the verfex and the propagator and some form of its derivative.

Even though, by general field theoretical arguments, the simple Green's functions obey certain well-defined, standard but formal equations, i.e. the "DysonSchwinger" equations, modulo the topology of the graphs characterizing a given theory; these equations are, in most cases, of very little practical analytical use. To arrive at a useful set of differential equations, which together with the relevant boun... dary conditions will yield a finite theory to all orders, one must operate on the formul equations in a manner which depends critically or the forn of the theory and its characteristic properties (for example, in QED gauge invariance dictates strongly the form 
of the renormalization recipe). As we will demonstrate later on, the invariance of the $\varphi^{4}$ interaction under General Linear Coordinate transformations plays a fundamental role in obtaining the equations for the propagator. Furthermore, the construction of the vertex function equations will be motivated by the particular form that the vertices take in this theory. In particular, we call all those O.P. I. vertices which can be split by cutting two internal lines Two-Particle Reducible (T.P.R.). All the others will be called Two-Particle Irreducible (T.P.I.) (see Fig. 4). The importance of this separation lies in the fact that the two-particle loop of the T.P. R. vertex graph exposes explicitly the primitive logarithmic divergence. Since the vertex, in this theory, is dimensionless, the integration over the loop is $\sim \int \frac{\mathrm{dk}^{2}}{\mathrm{k}^{2}}$ (within logarithmic terms). On the other hand, every sub-integration of the T.P. I vertices is convergent, since, from Fig. 4 for example, the six-point function goes as $\sim \frac{1}{k^{2}}$ and therefore both sub-integrations go as $\sim \int \frac{\mathrm{dk}^{2}}{\mathrm{k}^{4}}$. Of course the overall integral is still logarithmically divergent. This separation of T.P.R. and T.P. I. vertices becomes more striking if one realizes, as we will argue later on, that the primitive T.P. I. vertices have an overall single log. divergence, which can be handled very easily.

With this short summary of properties and nomenclature of relevant object of our theory, we will proceed to obtain the desired differential equations. First we will assume that the propagator function is known and we will renormalize the vertex. Then the program for the propagator will be carried out. This will eventually lead to the renormalization of the energy-momentum tensor. 
Chapter II

Renormalization of the Vertex Function

\subsection{Definition}

For simplicity, we find it convenient to study only the external leg-iruncated four-point Green's function which we express in coordinate space by $T\left(x_{1}, x_{2}, x_{3}, x_{4}\right)$ and which we will henceforth call the vertex function. From a more physical point of view, the Fourier transform of $T\left(x_{1}, x_{2}, x_{3}, x_{4}\right)$ can be looked at as a two-body, off-shell, scattering amplitude in the sense that

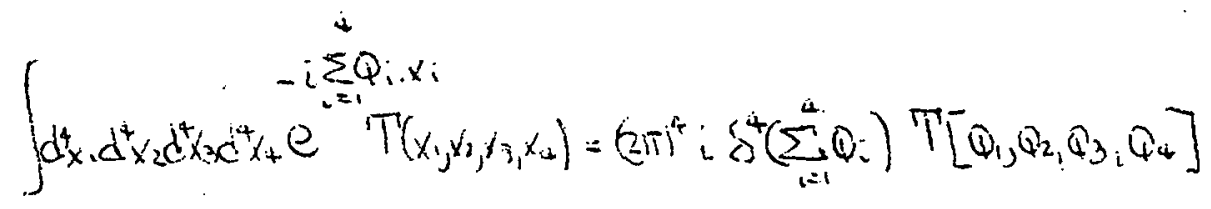

where the momentum space invariant amplitude

$$
\Pi\left[Q_{1}, Q_{2}, Q_{3}, Q_{4}\right]=T\left[S_{1} t_{1} \cup j Q_{1}^{2}, Q_{3}^{2}, Q_{3}^{2}, Q_{4}^{2}\right]
$$

is given in terms of (Fig. 5) the invariants

$$
\begin{aligned}
& S=\left(Q_{1}+Q_{2}\right)^{2} \\
& t=\left(Q_{1}+Q_{4}\right)^{2} \\
& U=\left(Q_{1}+Q_{3}\right)^{2}
\end{aligned}
$$

and the external, off-shell masses $Q_{i}^{2}$, satisfying the constraint

$$
S+t+\omega=\sum_{i=1}^{4} Q_{i}^{2}
$$




\subsection{Determination of the Formal Equations}

As we have already mentioned, we can separate the vertex function into two distinct parts, T.P.R. and T.P.I. vertices. The T.P.R. vertices involve only vertices connected by two-particle cuts, the integration over the two-particle loop giving rise to the primitive log. divergence of the theory. The T.P. I. vertices involve three or more particle cuts connecting four- and higher point functions. We will call these contributions to the vertex the V-graphs. (see Fig. 6). With separation in mind, and requiring crossing symmetry, T will obey a set of coupled Bethe-Salpeter type "Dyson-Schwinger" equations, described graphically in Fig. 7, which we represent symbolically as

$$
\begin{aligned}
& \left.T=K_{s}+\frac{1}{2} K_{s}(\Delta \Delta)_{s} T=K_{s}+\frac{1}{2} T \Delta \Delta\right)_{s} K_{s} \\
& K_{s}=V+\frac{1}{2} K_{t}(\Delta \Delta)_{t} T+\frac{1}{2} K_{v}(\Delta)_{s} T \\
& K_{t}=V+\frac{1}{2} K_{v}(\Delta \Delta) T+\frac{1}{2} K_{s}(\Delta \Delta)_{s} T \\
& K_{v}=V+\frac{1}{2} K_{s}(\Delta \Delta)_{s} T+\frac{1}{2} K_{t}(\Delta \Delta)_{t} T .
\end{aligned}
$$

The symbolic and graphical notations will be used, as dictated by necessity and/or convenience, interchangeably. Equation (2.5) (or Fig. 7) will generate the appropriate graphical solution (see Fig. 8), although, as we will argue further on, it is of little quantitative value. The Bethe-Salpeter kernels, $K_{s}, K_{f}, K_{u}$ are defined to be contributions to $T$ which are T.P. I. only in the $s, t, u$ channels respectively. By $\Delta$, we mean the full propagator. The factor of $1 / 2$ is required by the correct counting of graphs and it is a characterization of the symmetries of the theory $(1)$. 
If should be remarked that $(2.5)$ is a general formal equation for two-body, off-shell scattering amplitudes ${ }^{(20)}$. Appropriate dynamical theories will determine specific values for the "propagator" $\Delta ;$ the factor $1 / 2$ will be replaced by some suitable parameter. By construction, (2.5) obeys explicitly two-particle unitarity. The important element in our separation is the T.P. I. in every channel contribution, the $V$-graphs. Since $V$ acts as the driving term of the "integral equation" for $T$, it is important that, if some reduced, valid analytical form of (2.5) is to be eventually used for computational purposes, it must have some special, simplifying properties. This is the case for our interaction. First, $V$ has the property that its skeletons (or primitive parts) are divergent at most as a single power of a logarithm. A simple, arbitrary differentiation will reduce $V$ to its finite form. The nature of this restricted form of divergence can be traced from studies of the analytic properties of the $V$ graphs or more directly by analysis of their characteristic structure (in $\varphi^{4}$ theory) in coordinate space $^{(10)}$. The second property of $V$ makes (2.5) a simple and compact as possible equation for $T$ in terms of itself and the propagator only. As we have already seen, since $V$ is T.P.I. in every channel it is constructed from the higherpoint functions of the theory. We see then no point in working with such functions ${ }^{(5)}$ and the complicated equations which define them. (Of course, our only loss is that we do not have a closed form for $V$, which is, anyway, unessential to the renormalization construction.)

\subsection{Renormalization}

Recognizing that (2.5) is only a valid graphical equation for the vertes, we will now reduce it to a finite differentiated form. If we start by assurning that all functions 
in $(2.5)$ correspond to the unrenomalized ones, we notice that if we define how functions $V_{R}, T_{R},\left(K_{s, t, u_{R}}\right)$ and $\Delta_{R}$ by

$$
\begin{aligned}
& \Delta_{R}=Q^{-1} N \\
& T_{R}=R^{2} T \\
& \dot{f}_{R}\left(T_{R}\right)=V(T) R^{2} \\
& \left(K_{s+4 u}\right)_{R}=R^{2}\left(K_{-s, t u}\right)
\end{aligned}
$$

where $R$ is some arbitrary constant (which may or may not be infinite), then we observe that these new R-functions obey exactly the some equation (2.5) as $T, \Delta$, $K, V$ independently of the nature of $R$, except for the point term of $V$ which will be proportional to $R^{2}$. In particular, $(2.5)$ can be understood to represent formally, within an arbitrary constant term, the renomalized vertex and propagator. Now if $T$ and $\Delta$ are to be the renormalized functions, then the kernels $K_{s}, K_{f}, K_{u}$ must be undefined, logarithmically divengent functions due to both the ambiguity in the constant V-term and the bad convergence properties of the two-particle loop integrals. Our reduction procedure must eliminate the constant V-term ambiguity, and generate a new equation independent of the kernels. For this purpose, let us differentiate (2.5), without specifying the nature of the differential operator yet, to obtain an equation. for the differentiated vertex $\mathrm{T}^{\prime}$ (we will denote differentiated functions by "priming" them) such that

$$
\begin{aligned}
& T^{\prime}=k_{s}^{\prime}+\tau_{s}^{\prime}\left(k_{s}, \Delta_{,} \Delta^{\prime}, r^{\prime}\right) \\
& k_{s}=\dot{v}^{\prime}+\dot{\tau}_{t}+\dot{\tau}_{v} \\
& k_{t}=\dot{v}+\frac{1}{\tau} v+\frac{1}{\tau_{s}} \\
& k^{\prime}=v^{\prime}+t_{s}+t_{t}
\end{aligned}
$$


A simple operation shows that form $(2.7)$ can always be obtained from $(2.5)$, independently of the differential operator. The function $\tau_{s}^{\prime}$ is given by (see Fig. 9)

$$
\begin{aligned}
& \dot{\tau}_{s}=\frac{1}{2} T(\Delta \Delta)_{s}^{\prime} T+\frac{1}{2} k_{s}(\Delta \Delta)_{5} T+\frac{1}{2} T(\Delta \Delta)_{s} k_{s}+ \\
& +1 / 4 T(\Delta \Delta)_{s} K_{s}(\Delta \Delta) T
\end{aligned}
$$

with analogous expressions for $\tau_{+}^{\prime}, \tau_{u}^{\prime}$. If the differential operator has been properly chosen, $V^{\prime}, K_{s}^{\prime}, K_{t}^{\prime}, K_{u}^{\prime}$ and $\tau_{s, t, u}^{\prime}$ will be finite and therefore one can calculate by iterating $(2.7)$ and $(2.8)$ the differentiated vertex $T^{\prime}$. The full vertex function $T$ will be obtained by doing a suitable integration. To do this in a unique way, we must specify a boundary condition on $T$. This will be done by specifying $T$ at its symmetry point to be equal to the renormalized, physical coupling, constant $\mathbf{g}$ :

$$
\left.T\left[S, t ; Q_{1}^{2}, Q_{a}^{2}, Q_{3}^{2}, Q^{2}\right]\right|_{\text {symm pt. }}=\theta
$$

From our kinematics, the symmetry point can be specified by those momenta $Q_{i}^{5}$, $i=1,2,3,4$, such that

$$
\left(Q_{i}^{s}+Q_{j}^{s}\right)^{2}=\frac{4}{3}\left(1+2 \delta_{i j}\right) m^{2}
$$

where $m$ is the physical mass (which is known since the propagator is assumed knoivn). If $(2.7)$ and $(2.8)$ are symbolically rewritten as 
16.

$$
\dot{T} \equiv D_{\phi p} T \text {. }
$$

then

$$
T=g+\int_{D o p}^{1}
$$

where

$$
0=\left[\int_{D_{o p}}^{1}\right]_{\text {sum } p t .}
$$

We see then that, so far, we have completely avoided the notion of renormalization constants; we hove only noticed that (2.5) is, within an arbitrary constant, not present in (2.7), invariant with respect to multiplicative renomal izations. In (2.7), (2.8) $V^{\prime}$. will start acting as a driving ter only fo fourth order toroondition (2.9) . . nd R. 12) will specify

$$
T^{(1)} \equiv g
$$

Because of its properties, $\mid V^{\prime} I_{\text {skeletons }}$, will be finite independently of $\mathbb{o p}_{\text {op }}$ " and furthermore, since all divergences are basically logarithmic, any first order differential operator should make $\tau_{s}^{\prime}, \tau_{y}^{\prime}, \tau_{y}^{\prime}$ and $V^{\prime}$ finite. The existence of the well known overtopping divergences will limit our choice of $\delta_{\text {op }}$. We find that the simplest and most effective differential operator that resolves the overlaps in (2.8) is Ward's ${ }^{(22)}$ scaling derivative

$$
\Phi_{S} \equiv \sum_{i=1}^{4} Q_{i} \frac{\partial}{\partial Q_{i}}
$$


which has the property that, if we define for any function of the momenta $Q_{i}$

$$
A_{(}\left(Q_{1}, Q_{4}, Q_{3}, Q_{4}\right) \equiv Q_{S} A\left(\psi_{1}, Q_{7}, Q_{3}, Q_{4}\right)
$$

then

$$
A^{\prime}\left(\omega_{1}, Q_{2}, Q_{3}, \psi_{4}\right)=\left.\lambda \frac{d}{d \lambda} A\left(\lambda \omega_{1}, \lambda \dot{\alpha}_{1}, \lambda \psi_{2}, \lambda \psi_{4}\right)\right|_{\lambda=1}
$$

This equation implies that every internal line of a given graph to be differentiated on by $\boldsymbol{\theta}_{5}$ will be treated on an equal basis by its operation, being independent of the $Q_{i}$ 's, so that, with minimal consistency, one can choose convenient internal momenta routings and thus avoid the tedious and cumbersome task of defining rules for "directional derivatives" for each individual set of graphs ${ }^{(1),(5)}$. Using (2.7), (2. 12) will become

$$
\Pi=g+\int_{0}^{i} \frac{d \lambda}{\lambda}\left[\Gamma^{\prime}\left(\lambda Q_{i}\right)-\dot{\Gamma}\left(\lambda \psi_{i}^{s}\right)\right]
$$

where the momenta $Q_{i}^{s}$ obey (2.10).

The renormalization program for the vertex can be considered finished. It is summarized by equations $(2.7),(2.8),(2.9),(2.17)$ and $(2.18)$. So, assuming $\Delta$. to be known, these equations yield, by iteration, a finite result for $T$, to all orders in $\mathbf{g}$. 
18.

Chapter III

Renormalization of the Propagator

3.1 Preliminaries

As we have mentioned before, the propagator function of this theory is

primitively quadratic divergent. : Our scope is to obtain from a formal defining: "DysonSchwinger" equation a second-order differential equation which, together with two appropriate boundary conditions, can be solved to give a unique finite result.

The first step will be; in a manner very much analogous to the treatment of the vertex function, to take some sort of scaling derivative of the formal equation. This, if properly done, will get rid of the overtopdigg divergences; and if the right functions are studied, the degree of divergence will be lowered only to an overall logarithmic one which can then be taken care of by some other differentiation. As it turns out, what dictates the elements of this first step-proper scaling derivative, proper functions to apply such oscoling derivatyeds is underlying symmetry principle, characteristic of the interacticn under consideration. For example; in QED a successful program for the electron propagator is constrained by the gauge invariance requirements as manifested through the use of Ward's identity, which effectively represents a zero-energy photon insertion of the propagator (which is equivalent to taking a scaling derivative of the propagator). Of course, our interaction is physically simpler than QED; we don't have a gauge-invariance condition to impose; so we have to look for some other larger, but nonetheless basic, regulating symmetry. The obvious choice is the invariance of our interactioniunder arbitrary Lineareoordinate Transformations (L.C.T.), which we discuss later on. In analogy 
to QED, one should find a corresponding "Ward Identity", which in this case will represent insertions of zero-energy (long-wavelength) "gravitons" into the propagator. This is due to the fact that the arbitrary L. C.T. Ward Identify is of tensor nature. We will observe that the imposition of tracelessness to the resulting tensor form of the propagator is crucial in making certain integrals converge, much in analogy, again, to the imposition of the gauge condition in QED.

\subsection{Invariance under Arbitrary L.C.T. and the "Ward Identity"}

The removal of the overlapping divergences from the propagator graphs is quite standard; we must just vary the rechnique to accommodate our "graviton" insertion concept. As usual, let us define the (unrenormalized) propagator by the "DysonSchwinger" formal functional equation

$$
\Delta^{-1}\left(Q^{2}\right)=q^{2}+m_{0}^{2}+\sum^{*}\left(q^{2} ; \Delta\right)
$$

where $m_{0}^{2}$ is the unrenormalized mass, and $\Sigma^{*}\left(q^{2}, \Delta\right)$ represents the sum of all O.P.I. self-energy graphs (Fig. 10) and it depends functionally on the propagator. For convenience, and to justify certain formal manipulations, let us make $(3.1)$ finite by introducing a cut-off $A$, which we assume to be large, but finite

$$
\Delta_{\Lambda}^{-i}\left(q^{2}\right)=q^{4} / \Lambda^{2}+q^{2}+m_{0}^{2}+\sum^{*}\left(q^{2}, \Delta_{\Lambda}\right)
$$

Let us now exploit the invariance of the interaction under arbitrary linear coordinate transformotions. Let 
20.

$$
x_{\mu} \rightarrow x_{\mu}^{\prime}=\rho^{\mu v} x_{\nu}
$$

where $p$ is some arbitrary, constant matrix, def $p \neq 0$. Then the interaction density

$$
H_{I} \equiv \int d^{4} x Q^{4}(x)=\text { invariant }
$$

if the field transforms as

$$
\varphi(x) \rightarrow(\operatorname{det} \rho)^{-\frac{1}{4}} \varphi(\rho x)
$$

It is convenient to work in momentum space, where the above transformation will be equivalent to

$$
P^{\mu} \rightarrow P^{i \mu}=\lambda_{\mu \nu} P^{\nu}
$$

From (3.5) we can determine the transformation properties of the propagator, and so we can obtain the "Ward Identity" which defines the zero-energy graviton insertion tensor vertex

$$
\theta^{l \nu}(P, P)=-\frac{1}{\partial} \frac{\partial}{\partial \mu \nu}\left[(\operatorname{det} \lambda)^{-1 / 2} \Delta^{-1}(\lambda P)\right]_{\lambda=I}
$$

We do the explicit differentiation, recalling that

$$
\Delta[\log (\operatorname{det} \lambda)]=S \lambda^{\nu} x_{\mu \nu}^{-1}
$$


21.

and that therefore

$$
\left.\frac{\partial(\operatorname{det} \lambda)}{\partial \lambda^{u v}}\right|_{\lambda=I}=g^{\mu \nu}
$$

The Ward Identity will read, explicitly

$$
E^{\mu v}(p, p)=-\frac{1}{\partial} p^{\nu} \frac{\partial}{\partial p^{\mu}}\left[S^{-1}(p)\right]+14 g^{\nu} \Delta^{-1}(p)
$$

By general invariance arguments, we can decompose the tensor $\theta^{\mu \nu}(P, P)$ as follows

$$
\theta^{\mu \nu}(p, P)=p^{4} p^{N} \theta_{1}(p)+1 / 4 g^{2} \theta_{a}\left(p^{2}\right)
$$

Comparing (3.11) to $(3.10)$, we see that the form factors $\theta_{1}$ and $\theta_{2}$ obey the relations

$$
\begin{aligned}
& \theta_{1}\left(p^{2}\right)=-\frac{\partial}{\partial p^{2}}\left[\Delta^{-1}\left(p^{2}\right)\right] \\
& \theta_{2}\left(p^{2}\right)=\Delta^{-1}\left(p^{2}\right)
\end{aligned}
$$

These equations imply that if $\theta_{1}$ is known then the propagator $\Delta$, and therefore $\theta_{2}$. can be determined by a simple integration. Another decomposition of $9^{\text {th }}$ is of interest, mainly its separation between its traceless and nonutracelatss parts

$$
\begin{aligned}
& \theta^{\mu \nu} \equiv \theta^{\mu \nu}-1 / 4 g^{\mu \nu} \operatorname{Tr}\left(\theta^{\mu}\right) \\
& \operatorname{Tr}_{r}^{\mu \nu}=0
\end{aligned}
$$


Equation (3.11) yields

$$
\begin{aligned}
\bar{\theta}^{\mu \nu}(P, P) & \equiv P_{\mu v} \theta_{1}\left(P^{2}\right)= \\
& =\left(P^{N} P^{N}-1 / 4 \delta^{4} P^{2}\right)\left(-\frac{\partial}{\partial P^{2}} S^{-1}\left(P^{2}\right)\right)
\end{aligned}
$$

In conclusion, a determination of the traceless part of the fensor vertex will also determine both its trace and, therefore, its full form

$$
\begin{aligned}
\operatorname{Tr} \theta^{k \nu} & =P^{2} \theta_{1}\left(p^{2}\right)+\theta_{0}\left(p^{2}\right)= \\
& =-p^{4} \frac{\partial}{\partial p^{2}}\left(\Delta\left(p^{2}\right) / p^{2}\right)
\end{aligned}
$$

Interestingly enough, we will next show that, in fact, only $\Phi^{\mu \nu}(P, P)$ can be computed explicitly by reducing (3.2) to a finite equation. Equivalent terms for the trace or the full tensor cannot be determined in closed form.

\subsection{The Renormalized Equations for. $\Delta$}

Let us now investigate what kind of equation is obeyed by $\theta^{\mu \nu}(P, P)$. We will use (3.7) on the cut-off propagator defined by (3.2). With a finite cut-off all quantities are finite. Eventually the cut-off will be made infinite and if the right reduced equations are obtained, they will be well defined and finite in this limit. Doing the arbitrary linear transformation on (3.2) we get 
23.

$$
\begin{aligned}
\frac{1}{(\operatorname{det} \lambda)^{1 / 2} \Delta_{\mu}(\lambda P)} & =\frac{\left(\lambda^{\mu \alpha} P_{\alpha} \lambda^{\mu \rho} P_{\beta}\right)^{2}}{(\operatorname{det} \lambda)^{1 / 2} \Lambda^{2}}+\frac{\left.\left(\lambda^{\mu \alpha} P_{\alpha}\right)^{\mu \beta} P_{\beta}\right)}{(\operatorname{det} \lambda)^{1 / 2}}+ \\
& +\frac{m_{0}^{2}}{\left(\operatorname{det} \lambda \lambda^{1 / 2}\right.}+\frac{1}{(\operatorname{det} \lambda)^{1 / 2}} \sum^{*}\left(\lambda \rho_{j} \Delta_{1}(k)\right)
\end{aligned}
$$

The $k$ in $\Sigma^{*}$ represents the internal momenta which will be integrated on. As a direct consequence of the invariance of the interaction under arbitrary L. C.T., $\Sigma^{*}$ has the following, crucial property

$$
(\operatorname{det} \lambda)^{-1 / 2} \sum^{*}\left(\lambda P_{1} \Delta_{x}(k)\right)=\sum^{+*}\left(P_{1}(\operatorname{det} \lambda)^{1 / 2} \Delta_{\lambda}(\lambda k)\right)
$$

that is, the transformation on the external momentum $P$, effectively becomes a transformation on the internal variable momenta. Upon differentiation with respect to $\lambda^{\mu \nu}$ every internal propagator will be treated on the same footing, independently of the external momenta. This is the condition that allows for the elimination of the overlapping divergences.

Explicit differentiation yields

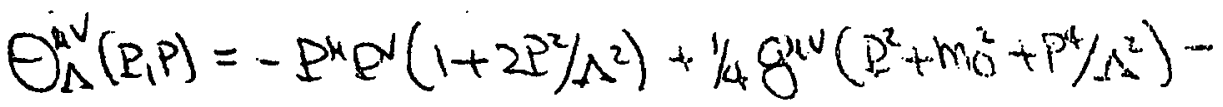

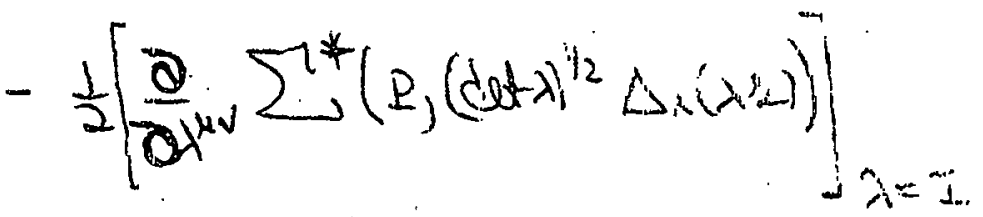


The term in brackets can be rewritten as an integral

$$
\begin{aligned}
& \frac{i}{2} \frac{\partial}{\partial x^{2}} \sum^{*}\left(P,(\operatorname{dot} \lambda)^{1 / 2} \Delta n(\lambda k)\right)= \\
& =\int \frac{d+k i}{(2 \pi)^{4}} \frac{1}{2} \frac{\left.\partial[\operatorname{det} \lambda)^{1 / 2} \Delta_{\lambda}(\lambda k)\right]}{\partial \lambda^{4 v}} \frac{\delta \sum^{*}\left(P_{1}(\operatorname{det} \lambda)^{1 / 2} \Delta_{n}(\lambda k)\right)}{\delta\left[(d e t \lambda)^{1 / 2} \Delta_{n}\left(\lambda_{k}\right)\right]}
\end{aligned}
$$

The operation $\frac{\partial \Sigma^{*}}{\partial \Delta}$ is essentially the responsible one in removing the overlapping divergences. Note that this object is less divergent than $\Sigma^{*}$ since one is effectively cutting open an internal propagator by the action $\frac{\partial}{\partial \Delta}$. More specifically we have ${ }^{(23)}$

$$
\frac{\delta \Sigma^{*}}{S \Delta}=\frac{1}{2} K_{s}\left(k, p_{,}-p_{1}-k\right)
$$

where $K_{s}$ has been defined by (2.5). The factor $1 / 2$ assures proper counting. Using (3.7) to express the other factor of the integrand in (3. 19) in terms of $\theta^{\mu \nu}$, we obtain the following integral equation (see Fig. 11)

$$
\begin{aligned}
& \theta_{\Omega}^{\mu^{J}}(P, P)=\left(\theta_{\Lambda}^{\mu \nu}\right)_{0}+ \\
& +\frac{1}{2} \int \frac{i d+k}{(2 \pi)^{4}} k_{S}(h, P) \Delta_{\Lambda}(k) \theta_{\mu}^{\mu \nu}(k, h) \Delta_{\Lambda}(k)
\end{aligned}
$$

Equation (3.21) has the property that, if we define new functions $\Delta_{\Lambda}^{R},\left(K_{S}^{A}\right){ }_{R}$. $\left(\theta_{\Lambda}^{\mu \nu}\right)_{R}$

$$
\begin{aligned}
& \Delta_{\Lambda}^{R}=R^{-1} \Delta_{\Lambda} \\
& \left(\theta_{\Lambda}^{\mu}\right)_{R}=R \theta_{\Lambda}^{\mu \nu} \\
& (K \hat{s})_{R}=R^{2} K_{S}^{\Lambda}
\end{aligned}
$$


where $R$ is some arbitrary constant, then these new functions will also obey it, within an undefined homogeneous term given by

$$
\begin{aligned}
& \left(\Theta_{\Lambda}^{\mu^{\nu}}\right)_{R}^{0}=Q\left(\Theta_{\Omega}^{\mu j}\right)^{0}= \\
& =R\left[P^{r} \rho^{v}\left(-1-2 P^{2} / \Lambda^{2}\right)+1 / 4 \lambda^{\mu v}\left(P^{2}+m_{0}^{2}+P^{4} / \Lambda^{2}\right)\right]
\end{aligned}
$$

In this sense, we expect that the reduced, finite form of $(1.38)$ can be written in terms of the renormalized functions, as $\Lambda$ approaches infinity. In terms of (3.23), (3.21) can be considered to be the formal equation for the renormalized propagator. Of course, a first condition on the reduction differential operator that will make (3.21) finite is that it must annihilate the undefined homogeneous term. The integral in $(3.21)$ is both divergent and undefined since it contains the undefined kernel $K_{s}$. Nevertheless, in accordance with our provisions, the equation that will give us, when suitably differentiated, a finite cut-off independent result, must have a simple overall log. divergence. To see how one can extract this behovior from (3.21), we must analyze the divergence structure of its integrand. For this purpose we define an integral

$$
\begin{aligned}
& J_{\Lambda}\left(f^{x}\right)=\frac{p^{r} p^{\nu}}{P^{4}} \int d k K_{s}^{\Lambda}\left(f_{1} k\right) \Delta_{\Lambda}(k) \theta_{\Lambda}^{\mu \nu}(k, k) \Delta_{\Lambda}(k) \\
& \equiv \int d x^{2} I_{\Lambda}\left(t^{2} j p^{2} ; m^{2}\right)
\end{aligned}
$$


where

$$
I_{\lambda}\left(k^{2}, p^{2}, m^{2}\right) \equiv\left\langle h^{c} K_{S}^{\Lambda}(k, p) \Delta_{n}(k) \frac{p^{\mu} E^{\mu \nu} p^{\nu}}{p^{4}} x_{n}(n)\right\rangle_{k}
$$

The bracket means that we are taking an angular average over the direction of the internal four-momentum $k$. The integral in $(3.24)$ is to be understood as being a Wick-rotated, Euclidean one. There are two regions in which we can analyze $J_{\wedge}\left(P^{2}\right)$, $k>\Lambda$ and $k<\wedge$, so that

$$
J_{\infty}\left(p^{2}\right)=\int_{0}^{\infty} d k^{2} I_{\infty}\left(k^{2}, p^{2}, m^{2}\right)+\lim _{\Lambda \rightarrow \infty} \int_{\lambda^{2}}^{\infty} d k^{2} I_{\Lambda}\left(k^{2}>\Delta^{2} j p^{2}\right)
$$

By $1_{\infty}$, we mean its expression in terms of cut-off independent, renormalized functions. The nature of the divergence of $J_{\infty}\left(p^{2}\right)$ will be determined from the behavior of $1_{\infty}\left(k^{2}, p^{2}\right)$ as $k^{2} \rightarrow \infty$.

The kernel $K_{s}$ can be expanded in terms of the angular polynomiats defined by ${ }^{(24)}$

$$
\left(1+t^{2}-2 t z\right)^{-1}=\sum_{n=0}^{-\infty} C_{n}(z) t^{n}
$$

which obey the orthogonality rule

$$
\left\langle C_{m}\left(z_{k}\right) C_{n}\left(z_{n}\right)\right\rangle k_{k}^{\wedge}=S_{v r}
$$


When $k^{2}>p^{2}, m^{2}$, the expansion can be written as

$$
\lim _{l^{2} \rightarrow \infty} K_{S}(p, k)=\sum_{n=0}^{\infty} C_{n}(\hat{u}, \hat{p}) k_{n}^{S}\left(l^{2}>p^{2}\right)
$$

The function $k_{0}^{S}$ will contain, of course, an arbitrary divergent piece due to the ambiguity in the definition of $K_{S}$. Except for that term, which will tum out to be irrelevant anyway, all the other $k_{n}^{S_{1}}$ s can be determined from $(2.7)$ and (2.8). To all orders in perturbation theory $(10)$, one can show that, as $k^{2} \gg p^{2}, m^{2}$

$h_{0}^{S} \rightarrow$ INFINITE CONSTANT $+h_{0}\left(h^{4}\right)+p^{2} / h^{2}+H_{0}\left(l^{2} / p^{2}\right)+\left(Q\left(\frac{1}{l^{4}}\right)\right.$

$h_{2}^{s} \rightarrow p^{2} / h^{2}+b^{2}\left(h^{2}\right)+C\left(1 / k^{q}\right)$

$\mathrm{H}_{0}$ and $\mathrm{H}_{2}$ grow af most as some power of $\log k^{2}$, as $k^{2}$ is large.

From (3.11) and (3. 15) we have that

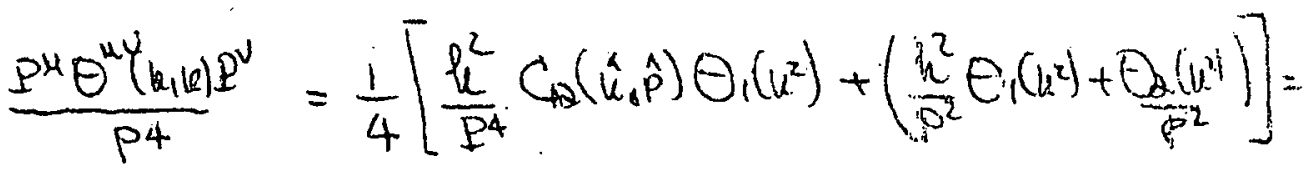

$$
\begin{aligned}
& =\frac{1}{4} \frac{h_{2}^{2}}{p^{4}} \theta_{1}\left(u^{2}\right) C_{0}\left(l^{4} \cdot p^{4}\right)+\frac{1}{4 p^{2}} T_{r} \cdot \Theta^{\left.x^{2}\right)}\left(u u_{1} u\right)
\end{aligned}
$$

Let us now decompose $I_{\infty}\left(k^{2}>p^{2}\right)$ into its $S(n=0)$ and $D(n=2)$ wove contributions, from $(3.30)$ we have 
28.

$$
\begin{aligned}
& \left.I_{\infty}\left(u^{2}\right\rangle p^{2}\right)=I_{\infty}^{s}+I_{-\infty}^{D} \\
& I_{\infty}^{S}=\frac{1}{4 p^{2}} \operatorname{Tr} \Theta^{4}\left(k_{1}, k\right)\left[h_{0}\left(l^{2}\right)+P^{2} / h^{2}+H_{0}\left(l^{2}, p^{2}\right)\right] \frac{1}{l^{2}} \\
& I_{O}^{D}=\frac{1}{4} H_{2}\left(k^{2}\right) \theta_{1}\left(k^{2}\right) \frac{1}{\ell^{2}}+O\left(1 / k^{4}\right)
\end{aligned}
$$

We then observe that the S-wave piece will diverge quadratically while the D-wave piece will only have an overall log. divergence $\left(\theta_{1}\left(k^{2}\right)\right.$ does not grow more than $\left.\log k^{2}\right)$. Since $(3.21)$ is linear in $\theta^{\mu N}, \bar{\theta}^{\mu N}$ the traceless part of $\theta^{\mu N}$ will also obey it. Obviously, because of (3.32) its integrand will only be D-wave, and therefore

$$
\int_{0}^{\infty} d x^{2} \frac{\partial}{\partial p^{2}} \overline{I_{\infty}}=\text { finite }
$$

and

$$
\frac{\partial}{\partial p^{\circ}} \lim _{\Lambda \rightarrow \infty} \int_{\Lambda}^{\infty} d x^{2} \bar{I}_{\lambda}=0
$$

Had we kept the full tensor (this also applies to its trace), $\int_{0}^{\infty} d k^{2} \frac{\theta}{\partial p^{2}} I_{\infty}\left(k^{2} p_{p}^{2}\right.$ )

would be infinite and $\frac{\partial}{\partial p^{2}} \lim _{\Lambda \rightarrow \infty} \int_{A}^{\infty} d k^{2} I_{A}\left(k^{2}, A^{2}, p^{2}\right)$ would contain the infinite piece necessary to cancel that one coming from $\frac{d_{\infty}}{\partial_{p}^{2}}$ (since the theary is renormalizable, the net result must be finite) plu! some unkmown contribution coning from evalum ating the integral at the upper limit and then letting $A \rightarrow \infty$. Even though, in proctice, this "surface" term cein be computed unicquely in an order-by-ordur prucess " 
this realization of the renormalization program is quite unexplicit and, in the light of what we have just discovered for $\sigma^{\mu N}(P, P)$, quite unnecessary.

Taking the limit as the cut-off becomes infinite, and after some algebra, we obtain the unique, finite integral differential equation for the renormalized form factor of the traceless part of $\theta^{\mu N}(P, P)$

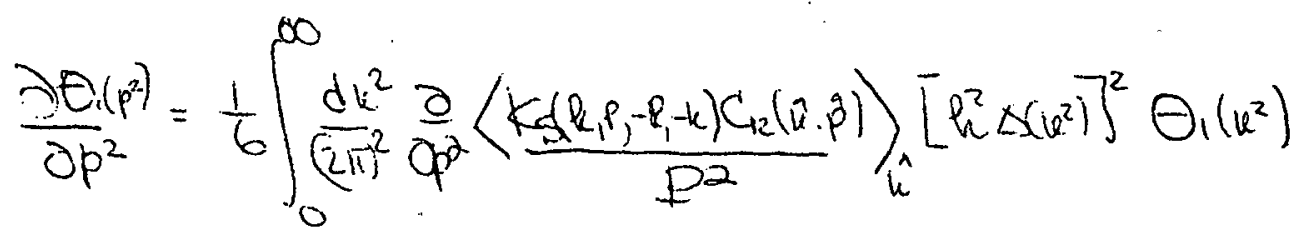

In order to integrate this equation so that we can use $(3.12)$ to find $\Delta\left(p^{2}\right)$ from a further integration, let us conveniently define

$$
\Phi_{1}\left(p^{2}\right) \equiv p^{2} \theta_{1}\left(p^{2}\right)=-p^{2} \frac{\partial}{\partial p^{2}}\left[\Delta^{-1}\left(p^{2}\right)\right]
$$

so that

$$
\Delta^{-1}\left(p^{2}\right)=-\int_{-m^{2}}^{p^{2}} \frac{d x}{x} \phi\left(x ; p^{2},-m^{2}\right)
$$

Here, we have used the boundary condition for the propagator

$$
\Delta^{-1}\left(-m^{2}\right)=0
$$

where now $\mathrm{m}^{2}$ is the physical mass. of the field. To get the boundary condition on $\phi_{1}\left(p^{2}\right)$, we note that in $(3.33)$ if we expand $\phi_{1}(x)$ by a power of $x$ about $x=0$, 
the leading term for $\Delta^{-1}$ will be $\sim-\int_{-m^{2}}^{p^{2}} \frac{d x}{X} \phi_{1}(0)=\phi_{1}(0) \log p^{2} /-m^{2}$, which implies a branch singularity at $p^{2}=0$. This is inconsistent with the analytic properties that one would like to have for a propagator function. Thus

$$
\phi_{1}(0)=0
$$

If we rewrite $(3.33)$ as

$$
\begin{aligned}
& \frac{\partial}{\partial p^{2}} \theta(p) \equiv \frac{I\left(p^{2} /-m^{2}\right)}{m^{2}} \\
& \frac{I\left(p^{2} / m^{2}\right)}{m^{2}}=\frac{1}{6} \int_{0}^{\infty} \frac{d u^{2}}{(2 \pi)^{2}} \frac{\partial}{\partial p^{2}}\left\langle\frac{k_{S} C_{p}}{p^{2}}\right\rangle_{\hat{k}}\left[l^{2} \Delta\left(k^{2}\right)\right]^{2} \theta(k)
\end{aligned}
$$

The $1 / m^{2}$ factor has been brought out to make $I\left(P^{2} /-m^{2}\right)$ a dimensionless function. From (3.38) and (3.37) $\phi_{1}\left(p^{2}\right)$ is calculated from

$$
\phi_{1}\left(p^{2}\right)=-p^{2} \int_{0}^{p^{2} /-m^{2}} d z I(z)
$$

As it turns out $(10)$, from the above equation (3.38), rogether with (3.35), ane cannot extract explicitly the asymptotic "zero-mass" form of the propagator functions. The relevant function in the asymptotic theory is net $Q_{1}\left(p^{2}\right)$ but the treces of $O^{(n)}\left(P_{8} P\right)$. From equation (3.15) we have

$$
T_{r} \theta^{\mu \nu}(p, p)=-p^{\alpha} \frac{\partial}{\partial p^{2}}\left[\frac{1}{p^{2} \Delta\left(p^{i}\right)}\right] \equiv i n(p-)
$$


One can then easily check that

$$
p^{2} \frac{\partial}{\partial p^{2}} \theta_{1}\left(p^{2}\right)=\frac{\partial \ln \left(p^{2}\right)}{\partial p^{2}}
$$

and therefore, using (3.34)

$$
\frac{\partial m}{\partial p^{2}}=-\frac{p^{2}}{G} \int_{0}^{\infty} \frac{d u^{2}}{\left(2 \pi^{2}\right.} \frac{\partial}{\partial p^{2}}\left\langle\frac{k_{s} C_{2}}{p^{2}}\right\rangle\left[\left.k^{2} \Delta\left(u^{2}\right)\right|_{\frac{\partial}{\partial k^{2}}}\left[s^{-1}\left(u^{2}\right)\right]\right.
$$

From $m\left(p^{2}\right)$ one can also easily determine, by integration, the propagator

$$
\left[P^{2} \Delta\left(p^{2}\right)\right]^{-1}=\int_{-m^{2}}^{P^{2}} \frac{d x}{x^{2}} m(x)
$$

The boundary condition for $m\left(q^{2}\right)$ is that

$$
\begin{aligned}
& m\left(p^{2}\right)=m^{2} \\
& \text { AT } p^{2}=-m^{2}
\end{aligned}
$$

where $\mathrm{m}^{2}$ is the physical mass. The vanishing of the trace of the "zero-mass" forward energy-momentum tensor at $p^{2}=0$ will be the key constraint in obtaining the asymptotic theory. Both sets of equations (3.38) with (3.35), and (3.43) with (3.42), will equally determine the renormalized propagator function, but only the latter has a smooth limit approaching the short distance region. The renormalization program is now complete. Equations (2.7), (2.8), (2.9), (2. 17), (2. 18), (3.35), (3.38) and (3.39), 
or $(3.42)$ and $(3.43)$, are a set of unique, coupled differential-integral equations which can be solved by iterations to yield the well known perturbation expansion to all orders in the renormalized coupling constant.

An explicit application of these simple and beautiful equations will be done in the following paper. There it is shown that the "zero-mass" theory of our interaction obeys analogous equations in terms of the "zero-mass" functions and appropriate boundary conditions. As a consequence one is also able to calculate explicitly all the relevant renormalization group parameters.

We will now extend the graviton insertion idea to the non-forward (non-zero energy insertion) case and thus we will obtain renor malized equations for the energymomentum tensor. 
Chapter N

The Energy-Momentum Tensor

4. 1 The Callan-Coleman-Jackiw Tensor

Recently, Collan, Coleman and Jackiw (18), while trying to study scale and conformal symmetry breaking, made some interesting remarks concerning the nature of the field energy-momentum tensor. First, they observed that if one adds a term proportional to $\left(\partial_{\mu} \partial_{\nu}-g^{\mu N} \square^{2}\right) \varphi^{2}$ to the canonical field tensor, such that

$$
\theta_{c c J}^{\mu v} \equiv \theta_{c}^{\mu \nu}-c_{0}\left(Q \partial v-g^{\mu v} \square^{2}\right) \varphi^{2}
$$

then the Poincaré structure of the theory will be left unchanged. Furthermore, it was observed that the particular choice $c_{0}=1 / 6$ will give

$$
\text { Tr } \theta_{c c J}^{\mu v}=m_{0}^{2} \varphi^{2}
$$

That is, the trace of the CCJ tensor will depend only on the operator $\varphi^{2}$, which has canonical dimensions less than four and would be called "soft". The relevance of this "softness" of the trace of the energy-momentum tensor to the breaking of scale symmetry (the close connection to conformal symmetry is lucidly discussed in (18) and (24)) stems from the fact that the generator of its transformations; $D^{\mu}$, is given by

$$
D^{\mu}=E_{c c J}^{\mu v} \times v
$$

such that

$$
\partial_{\mu} D^{\mu}=\operatorname{Tr}\left[\Theta_{c c J}^{\mu v}\right] .
$$


and would equal $m_{0}^{2} \phi^{2}$ for the $C C J$ tensor (partial conservation of the scale current). When an analysis of the corrections to (2. I) due to the interaction was made, CCJ thought that the characteristic value $c_{0}=1 / 6$ would still keep the trace soft to all orders and that it would give finite matrix elements of $\theta^{\mu N} \mathrm{Cl}{ }^{\circ}$

Within our program, we will study, to all orders explicisly, the renormallzation of the non-forward energy-momentum tensor by generalizing the results obtained while studying the renormalization of the propagator. We will determine shat the renormalio izations due to the interaction do require the addition ferm of (2. 1), but that no pars. ticular choice of the parameter $c_{0}$ will be singled out as special. In fact $c_{0}$ will be left undetemined by the renormalization procedure alone (this fact has al ready been observed by Symanzik $(7),(25)$. Furthernore the requirement of a "soft" trace will still leave $c_{0}$ undetermined since the trace cannot bo made soft untess one imposes some very stringent conditions that are beyond renomalization and perfurtication theory. (26).

\subsection{Definition ard the "Ward Identity"}

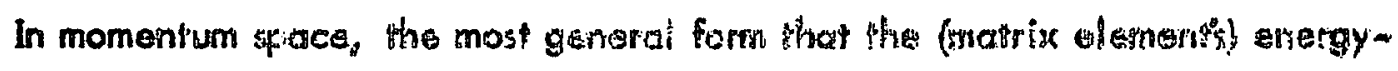
momentum fensor can toike is

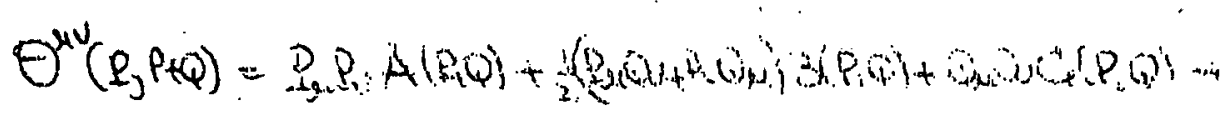

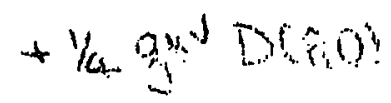

When $Q=0$, from Et apper 114, we ste that

$$
A(P, D)=-\frac{S}{S}|M(p)|
$$


and $A(P, 0)$ can be calculated from Eq. (3.33). This, with the help of Eq. (3.35), determines the propagator $\Delta\left(p^{2}\right)$. Equation (3.33) was obtained by exploiting the invariance of the interaction under general L.C.T. and the consequent renormalizability of the traceless forward tensor. The invariance under L. C.T. will be again exploited for the non-forward case. One will obtain a generalized L. C. T. "Ward Identity" which will be used to derive an integral equation analogous to $(3.21)$ for the traceless part of $\theta^{\mu N}(P, P+Q)$

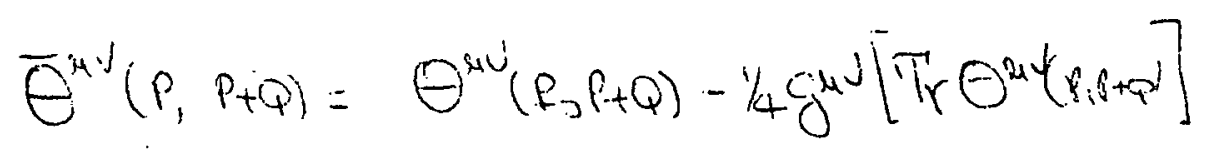

and thus it will help us determine the form factor $A(P, Q), B(P, Q)$ and $C(P, Q)$

$$
\begin{aligned}
& \bar{\theta}^{\mu \nu}(P, f+\psi)=\left(P^{\mu} p^{N}-1 / 4 g^{2} \rho^{2}\right) A(P, Q)+ \\
& +\frac{1}{2}\left(P_{2} Q_{3}+P_{v} \varphi_{2}-1 / 2 T^{N v} P(0) B(P, 0)+\right. \\
& +\left(G+1 / 40^{40} Q^{2}\right) \dot{G}(P, Q)
\end{aligned}
$$

The determination of the integral equation for $\bar{\theta}^{-N}$ is the bulk of this chapter and it will be discussed separately in the next section.

To compute the full tensor, $\theta^{\mu N}$, we must know its trace. This means that the $g^{\mu N}$ form factor, $D$, of $(4.5)$, left unspecified by $\bar{\theta}^{\mu N}$, must also be known. Its determination will be possible because of the "Ward Identity" that $\theta^{\mu N}$ must obey as a consequence of the Poincare structure of the theory

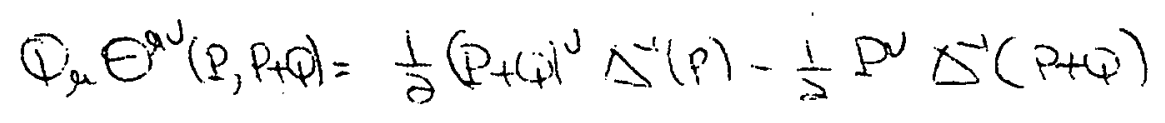


Because $\Delta(P)$ is known, the right-hand side of $(4.9)$ is known. Using $(4.5),(4.9)$ will imply that

$$
A(P, Q)(Q . P)+B(P, Q)\left(\frac{1}{2} Q^{2}\right)=\frac{1}{\partial}\left[S^{-1}(P)-S^{-1}(P+Q)\right]
$$

and

$\frac{1}{2}(P, \dot{Q}) B(P, Q)+\dot{\psi}^{2} G(P, \psi)+1 / 4 D=\frac{1}{2} \Lambda^{-1}(p)$

Equation (4. 10) just expresses the fact that $A$ and $B$ are not independent. If $A$ or B are known, then (4.10) will give the other. From (4.11) we can determine the trace (within "seagull" terms); Eq. (4.5) gives

$T_{r} \theta^{\mu \nu}=A(R Q) P^{2}+B(P, Q) P\left(\psi+Q^{2} C(P, Q)+D\right.$

so that, from $(4,11)$

$\operatorname{Tr} \theta^{m}=P^{2} A(P, Q)-B(P, Q)-3 C(P, Q) Q^{2}+2 S^{-1}(P)$

In zeroth order, because of $(4.10)$, we have

$$
\theta^{\mu v}(0)=-P_{\mu} P_{J}-\frac{1}{2}\left(P_{\mu} Q_{U}+P_{\nu} Q_{\mu}\right)+C_{0} Q_{\mu} Q_{V}
$$

where we let $c_{0}$ be undetermined. The trace becomes then 
37.

$$
\begin{aligned}
& \pi_{r}\left[\theta^{v}(0)\right]= \\
& \quad \frac{1}{\partial}\left(P^{2}+m_{0}^{2}\right)+\frac{1}{2}\left[(P+Q)^{2}+m_{0}^{2}\right]+m_{0}^{2}-\frac{1}{2} Q^{2}-3 Q^{2} C_{0}
\end{aligned}
$$

The first two terms are just seagulls and can be discarded. We notice that if $c_{0}=1 / 6$ the trace will be equal to $m_{0}^{2}$. This agrees with CCJ. Whether this will be true when the effects of the interaction are included will be discussed in the sections that follow.

4.3 Invariance under Arbitrary L.C.T. and the Formal Equations for $\sigma^{\text {N }}$

In Chapter III we saw that if we make the transformation of coordinates given by Eq. (3.3), the interaction will remain invariant if the field transforms as dictated by Eq. (3.5). A two-point function will then, in general, transform as

$$
\Delta x, y)=\left(\frac{\partial x}{\partial x^{\prime}}\right)^{\frac{1}{4}}\left(\frac{\partial y}{\partial y^{\prime}}\right)^{1 / 4} \Delta\left(x^{\prime}, y^{\prime}\right)
$$

So, if we write the equation analogous to $(3.10)$ in coordinate space

$$
\left.\Delta^{-1}(x, y)=\Delta_{0}^{-1}(x, y)+\sum^{*}(x, y) \Delta\left(z_{1}, z_{2}\right)\right)
$$

then, because, as can be easily checked, $\Sigma$ * has the property

$$
\begin{aligned}
\left(\frac{\partial x^{\prime}}{\partial x}\right)^{\frac{1}{4}}\left(\frac{\partial y^{\prime}}{\partial y^{\prime}}\right)^{\prime 4} & \Sigma^{t^{*}}\left(x^{\prime}, y^{\prime} j \Delta\left(z_{1} z_{2}\right)\right)= \\
& =\sum^{*}\left(x, y^{\prime},\left(\frac{\partial z_{1}^{\prime}}{\partial z_{1}^{\prime}}\right)^{\prime}\left(\frac{\partial t_{2}}{\partial t_{2}^{\prime}}\right)^{\prime / 4} \Delta\left(z_{1}^{\prime}, z_{2}^{\prime}\right)\right)
\end{aligned}
$$


one can carry out steps analogous to those that lead Eq. (3. 16) to Eq. (3.21), in coordinate space. As a consequence of these generalized "Ward identities", after doing a fourier transform, one gets the following formal "Dyson" equation for $\nabla^{\mu \mathrm{N}}$, the traceless tensor (the derivation that leads to this equation can also be done using standard field theoretical techniques) ${ }^{(27)}$

$$
\begin{aligned}
\bar{\theta}^{\mu \nu}(P, P+Q) & =\bar{\theta}^{\mu \nu}(P, P+Q)^{\nu}+ \\
+ & \int \frac{1}{2} \int \frac{d 4 k i}{(2 \pi)^{4}} k_{s}(-k, P,-P-Q, k+Q) \Delta(k) \bar{\theta}^{\mu \nu}(k, k+Q) \Delta(k+Q)
\end{aligned}
$$

This equation is depicted graphically in Fig. 12, and for convenience we will write it symbolically as

$$
\bar{\theta}^{\mu \nu}=\bar{\theta}_{0}^{\mu \nu}+\frac{1}{2} k_{s}(\Delta \lambda)_{s} \bar{\theta}^{\mu \nu}
$$

In agreement with the zeroth order colculation we can write

$$
\begin{aligned}
& \bar{\theta}_{0}^{\mu \nu}=-\left(P_{\mu} P_{\nu}-1 / 4 g \nu P^{2}\right)-\frac{1}{2}\left(P_{\mu} \varphi_{u}+P_{\nu} Q_{\mu}-\frac{1}{2} g \omega v P \cdot Q\right) \\
& +C_{0}\left(Q_{\mu} Q_{u}-1 / 48^{\ln } Q^{2}\right)
\end{aligned}
$$

with $c_{0}$ unspecified. The renormalization procedure should tell us something (or nothing) about its value. Obviously (4.19) is identical to the traceless form of (3.21) when $Q=0$ (within a mass factor is the homogeneous term). Equation (4.19) is, of course, only formal; calculations from it yield infinite results due to the ambiguity in the definition of $K_{s}$ and due to the poor convergence properties of the integrand. 
We must now determine an equation for a differentiated from of $\nabla^{-N}(P, P+Q)$ which will enable us to calculate, to all orders, finite form factors $A(P, Q), B(P, Q)$ and $C(P, Q)$.

\subsection{A General Equation for the Derivative of $\Xi^{\mu N}$}

Because of the non-trivial $P$ and $Q$ dependence of the integrand in $(4.20)$ our task of finding an equation for the derivative of $\bar{\theta}^{\mu N}$ will note be like the simple, one-step operation we were able to carry out for the $Q=0$ case. One of our primary concerns is to eliminate the ambiguous $K_{5}$ from our equations and replace it, as we did for the vertex equations, by its well-defined derivative. Using Eq. (2.5) defining $T$ in terms of $K_{s}$, we notice that (4.19) is equivalent to, symbolically (Fig. 13)

$$
\bar{\theta}^{\mu \nu}=\bar{\theta}_{0}^{\mu \nu}+\left.\frac{1}{\partial} T(\Delta x)\right|_{s} ^{\mu \nu}
$$

Denote differentiated functions by "priming" them. Then

$$
\begin{aligned}
\bar{\theta}^{\mu \nu}= & {\left[\frac{1}{\bar{\theta}^{\mu}}+\frac{1}{\partial} \pi_{0}\left(\Delta \nu_{s} \bar{\theta}_{0}^{\mu \nu}\right]+\right.} \\
& +\frac{1}{2} \phi(\Delta \Delta)_{s} \bar{\theta}_{0}^{\mu \nu}+\frac{1}{2} \pi(\Delta x)_{s}^{\prime} \bar{\theta}_{0}^{\mu \nu}
\end{aligned}
$$

The last two terms can be re-expressed in terms of $K_{s}^{\prime}, T$ and $\nabla^{\mu N}$, if we use (4.22) and (2.8). This gives the expression

$$
\begin{aligned}
\bar{\theta}^{\prime \nu} & =\left[\bar{\theta}^{\prime \mu}{ }_{0}+\frac{1}{\partial} T\left(\Delta \Delta_{s} \frac{1}{\bar{\theta}^{\mu}}\right]+\frac{1}{2} T\left(\Delta \Delta_{s}^{\prime} \bar{\theta}^{\mu \nu}+\right.\right. \\
& +\frac{1}{2} k_{s}^{\prime}\left(\Delta \Delta_{s} \bar{\theta}^{\mu \nu}+1 / 4 T \Delta \Delta\right)_{s} K_{s}\left(\Delta \Delta_{s} \bar{\theta}^{\mu \nu}\right.
\end{aligned}
$$


This equation is quite general and independent on the form of the differential operator defining $\bar{\theta}^{, \mu N}$. Recalling our discussion following (3.21), we must realize that if (4.24) is to be written in terms of renormalized quantities, the terms depending on $\bar{\theta}_{0}^{\mid N}$ will be left undetermined by an arbitrary constant multiplicity factor. To eliminate this indeterminacy we will impose on the reducing differential operator the condition that it must annihilate $\left(\bar{\theta}_{0}^{\mu \nu} R\right)$, where $R$ is the arbitrary multiplicative constant. In this case, (4.24) will become much simpler (Fig. 14) since the term in brackets will not be present.

If the reducing differential operator consists of differentials with respect to $P$ only, then (4.24) will reduce to the still simpler form

$$
\bar{\theta}^{\omega v}=\frac{1}{7} k_{B}(\Delta \Delta)_{s} \bar{\theta}^{\nu}
$$

with

$$
\frac{1}{\theta}^{\mu \nu} \equiv \theta_{p_{p}}(E) E^{\mu v}
$$

and

$$
\Phi_{\phi i} \theta_{0}^{m}=0
$$

since the integrands of the remaining terms vanish. Equation (4.25), plus the choice of $\mathscr{D}_{\text {op }}(\mathbb{P})$ which satisfies (4.27), represents, at least in principle, the solution to our problem.

To obtain a more explicit result, it is convenient to divide the problem into two separate parts. The reason to do so will become apparent as we carry through the 
41.

analysis. First, we will calculate $A(P, Q), B(P, Q)$ and $[C(P, Q)-C(O, Q)] \equiv \bar{C}(P, Q)$. Then we will independently determine $C(O, Q) \equiv C\left(Q^{2}\right)$. If we subtract from (4. 19) its $P=0$ part, we will obtain a new traceless tensor $\bar{\phi}^{\mu N}(P, P+Q)$

$$
\bar{\phi}^{\mu \nu}(P, p+Q) \equiv \bar{\theta}^{\mu \nu}(2, P+\psi)-\bar{\theta}^{\mu \nu}(0, Q)
$$

Then $\bar{\phi}^{\mu \nu}$ will have the same form factors $A$ and $B$ as $\bar{\theta}^{\mu \nu} ;$ but its $\left(Q^{\mu} Q^{\nu}-\frac{1}{4} g^{\mu \nu} Q^{2}\right)$ piece will be $\bar{C}(P, Q)$

$$
\begin{aligned}
& \vec{C}(P, \psi) \equiv C_{1}(P, \psi)-C_{1}(O, Q) \\
& \vec{G}(O, \psi)=0
\end{aligned}
$$

Analogously to $(4.25), \bar{\phi}^{\mu N}$ will obey an integral equation

$$
\frac{1}{\phi^{\mu v}}=\frac{1}{\partial}\left[K_{s}-K_{s}(f=0)\right](\Delta \Delta)_{s} \bar{\theta}^{\mu v}
$$

Note that because of Ward's identity $A(O, Q)$ and $B(O, Q)$, which will be used to specify boundary conditions, and $\bar{C}(O, Q)=0$, are known. Equation (4.25) and the Ward identity cannot specify $C(O, Q)$, because this would require knowing the trace of the full tensor $\theta^{\mu N}$. To determine $C\left(Q^{2}\right)$ we will have to use $(4.24)$ evaluated at $P=0$. The boundary condition on $C(O, Q)$ af $Q=0$ will specify the unknown $C C J$ constant $c_{0}$. 
4.5 Determination of $A(P, Q), B(P, Q)$ and $\bar{C}(P, Q)$

Equation (4.30) can be explicitly written as an integral

$$
\begin{aligned}
& \operatorname{QHe}_{p}(P) \bar{\phi}^{N}=
\end{aligned}
$$

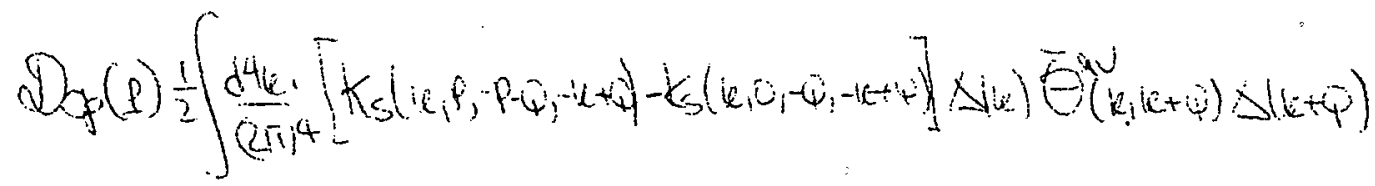

In order to determine which $\mathscr{O}_{\text {op }}$ makes (4.31) finite and obeys (4.27), we must analyze its integrand to see why it is divergent. The solution to the vertex equations in Chapter II indicates that the (finite, well-defined part of) kernel $K_{s}(k, P,-P-Q, k+Q)$ behaves, for large $k$ as

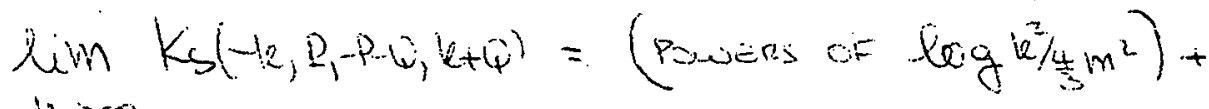

$$
\begin{aligned}
& r_{i} \rightarrow \infty \\
& +\left(\frac{P}{i k}\right) G(\hat{b}, \hat{p})+O\left(\frac{1}{i^{2}}\right)
\end{aligned}
$$

$C_{1}$ is the $P$-wave angular function defined by Eq. (3.27). The leading $k$-behavior of the subtracted kernel is then proportional to

$$
\left(E / l_{1}\right)(\hat{u} \hat{\phi})+Q\left(1 / e^{2}\right)
$$

Furthermore, because of the Ward identity, the form factors of $\sigma^{\mu N}$, if finite, have a large $k$-behavior dictated by the behavior of the propagator function. Then, as $k \rightarrow \infty$, the spectral representation of $\bar{\theta}^{\mu N}(K, K+Q)$, relevant to the angular 
43.

integration about $k^{\mu}$ of an integrand which depends on two different momenta, $P$ and $Q$, has the form

$$
\begin{aligned}
& \lim _{u \rightarrow \infty}\left[\bar{\theta}^{u v}\left(k_{i} \mid x+\bar{q}\right)\right]_{i \hat{k}}=
\end{aligned}
$$

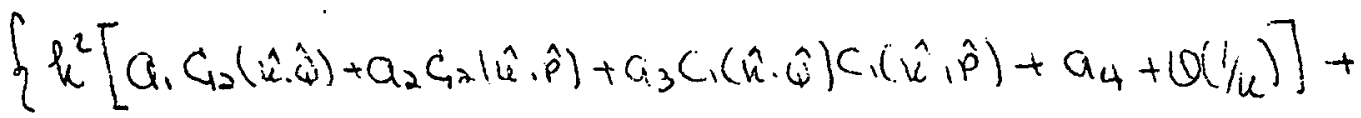

$$
\begin{aligned}
& \left.+k Q\left[a_{5} c_{1}(\hat{u} \cdot \hat{Q})+a_{6} c_{1}(\hat{k} \cdot \hat{p})+Q\left(k_{k}\right)\right]+a_{7} Q^{2}(1+0(1 / u))\right\}
\end{aligned}
$$

The $C_{1,2}$ are the $P$ and $D$ angular functions and the constants $a_{i}$ depends on the appropriate Clebsch-Gordon coefficients and structure of the form factors. The propagator $\Delta(k+Q)$ will behave as:

$$
\lim _{k \rightarrow \infty} \Delta(u+Q)=\frac{1}{k^{2}}\left[\sum_{n=0}^{\infty} C_{n}\left(\frac{Q}{k}\right)^{n}+0(1 k)\right]
$$

The angular functions, in addition to the rule given by Eq. (3.28), also obeys the important relations

$$
C_{m} C_{n}=\sum_{s=0}^{m} C_{n+m-2 s}
$$

$n$ TIm (SAME ANGLE)

and

$$
\left\langle\operatorname{Cm}(\hat{k} \cdot \hat{\psi}) C_{n}(\hat{k} \cdot \hat{p})\right\rangle_{k}=\frac{1}{n+1} \operatorname{Snm} \operatorname{Cn}(\hat{p} \cdot \hat{\psi})
$$


Using these facts, and (4.32), (4.33) and (4.34), it is easy to verify that the divergent part of the integral in (4.31) is of the form $\int^{\infty} \frac{d k^{2}}{k^{2}} \log ^{N} k^{2}$. Since the contribution of the subtracted kernel to this divergent integral is linear in. $P$, it will be annihilated by a simple double-p derivative. As a matter of fact, the differential operator

$$
D_{j p}(p) \equiv \frac{\partial}{\partial P^{*}} \cdot \frac{\partial}{\partial p^{*}}=D_{P}^{2}
$$

satisfies all our criteria, since

$$
\square_{P}^{2}\left(P_{\mu} P_{v}-1 / 2 \delta^{\mathrm{gv}} P^{2}\right)=0
$$

The finite expression of (4.31) is, then, of the form

$$
\begin{aligned}
& \prod_{P}^{2} \operatorname{T}^{2}(e, P+Q)= \\
& \left.=\frac{1}{2} \int \frac{d+k i}{(2 \pi, 4}\right]_{p}^{2}\left[k_{s}-\left(k_{s}\right)_{p=0}\right] \Delta(k) \bar{\theta}(k, k+Q) \Delta(k+\omega)
\end{aligned}
$$

This beautifully simple and compact equation represents, at least in principle, the solution to the first part of our problem. Unfortunately, its integration to determine $A, B$ and $\bar{C}$ is not so straightforward. Nevertheless, let us now show, for completeness' sake, that $A, B$ and $\bar{C}$ can be easily determined if, instead of choosing $\square_{p}^{2}$. as differential operator, the following slightly more complex one is considered

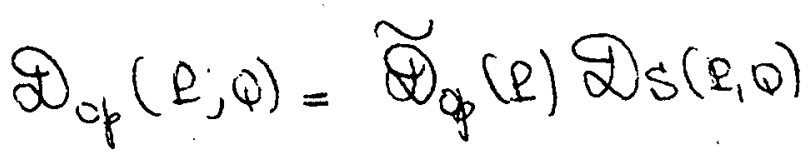


where

$$
\dot{\phi} s=\frac{1}{2}\left(P \cdot \frac{\partial}{\partial p}+Q \cdot \frac{\partial}{\partial p}\right)
$$

is the scaling derivative, and

$$
\tilde{H}_{\phi}(R)=P_{\alpha} f_{\beta} \frac{\partial}{\partial P_{\alpha}} \frac{\partial}{\partial P_{\beta}}=P \frac{\partial}{\partial P}\left(P \frac{\partial}{\partial p}-1\right)
$$

Because of the scaling derivative, if we apply $(4.40)$ to some function of $P$ and $Q$, we will have

$$
\begin{aligned}
& A(P, Q) \equiv P \cdot \frac{\partial}{\partial P}\left(P \frac{\partial}{\partial P}-1\right) \partial \mathcal{A} A(\rho, Q) \\
& =P \frac{\partial}{\partial P}\left(P \cdot \frac{\partial}{\partial p}-1\right)\left[\lambda \frac{d}{d \lambda} A\left(\lambda e_{1} \lambda Q\right)\right]_{\lambda=1} \\
& \equiv \mathscr{E}_{b p}(e) A_{s}\left(P_{1} Q\right)
\end{aligned}
$$

Since $\tilde{\mathcal{B}}_{o p}(p)$ and $\mathscr{E}_{S}(P, Q)$ commute, the scaled tensor function will obey an equation analogous to $(4.24)$

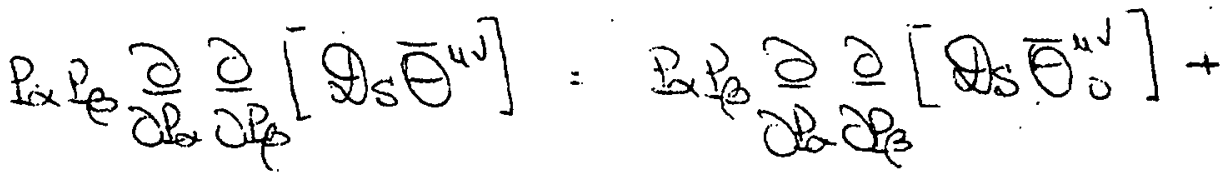

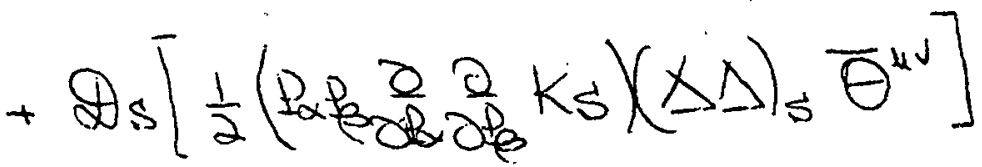


36

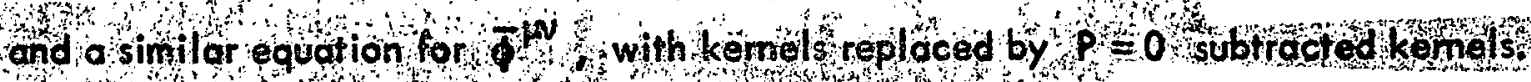

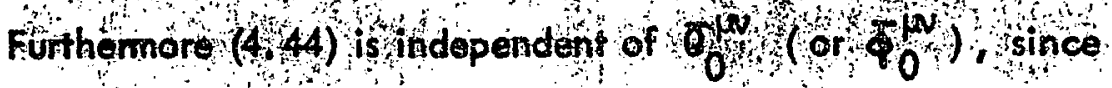

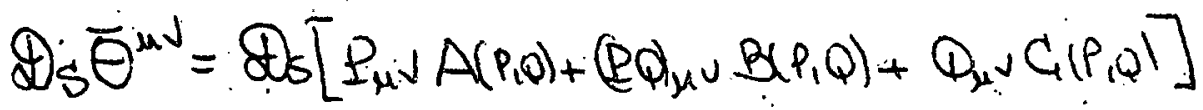

$$
\begin{aligned}
& =\bar{\theta}^{\mu \nu}+P_{\mu \nu} A_{S}(R, Q)+(P Q)_{\mu \nu} B_{S}(P, Q)+Q_{\mu \nu} C_{S}(R, Q) \\
& \equiv \bar{\theta}^{m \nu}+\bar{\theta}_{S}^{m v}
\end{aligned}
$$

(4.45a)

$$
\begin{aligned}
& P_{\mu \nu}=\left(P_{\mu} P_{\nu}-1 / 4 \delta^{\mu \nu} P^{2}\right) \\
& (P Q)_{\nu}=\frac{1}{2}\left(P_{\mu} Q+P_{\nu} Q_{\mu}-1 / 2 \delta^{\mu \nu} P Q\right) \\
& Q_{\mu \nu} \equiv\left(Q_{\mu} Q \nu-1 / 4 \delta^{\mu \nu} Q^{2}\right)
\end{aligned}
$$

and obviously

$$
\bar{\theta}_{0 s}^{\mu^{N}}=0
$$

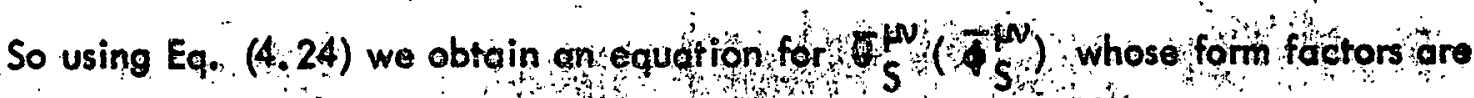
just the scaled form factors of of $\left(\frac{1}{\phi}\right)$, In toms of $\phi_{S}$

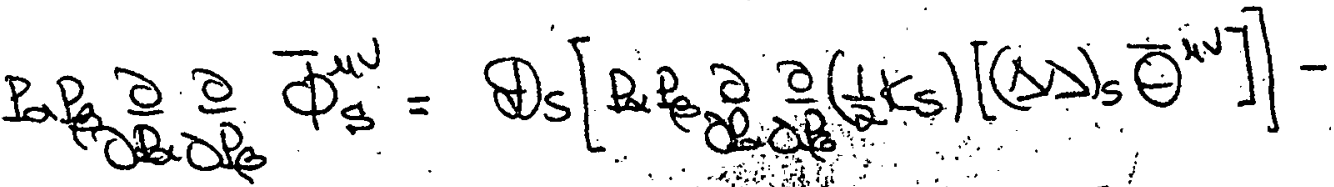

$$
\begin{aligned}
& \text { - Pa } \partial \partial\left(\frac{1}{2} L_{5}\right)\left[(\Delta x)_{5} \theta^{i \nu]}\right.
\end{aligned}
$$


Note that $P_{\alpha} P_{\beta} \frac{\partial}{\partial P_{\alpha}} \frac{\partial}{\partial P_{\beta}}$ has the right properties since the action (P. $\left.\frac{\partial}{\partial P}-1\right)$ will make the integrand convergent and the action of $P_{0} \frac{\partial}{\partial P}$ will eliminate the arbitrary infinite constant that comes with $K_{S}$. The determination of $A, B$ and $\bar{C}$ now follows immediately. First notice that

$$
J^{\mu V}(\rho, Q) \equiv\left\{P_{x} \frac{\partial}{\partial Q} \frac{\partial}{\partial P_{p}}\left[\frac{1}{2} k_{s}-\frac{1}{2}\left(k_{s}\right)_{R=0}\right]\right\}\left(\Delta \Delta_{s} \bar{\theta}^{u v}\right.
$$

is a well-defined, calculable integral which can be rewritten as

$$
J^{\mu \nu}(P, Q)=P_{\mu} J J_{1}(P, Q)+(P Q)_{\mu} J_{2}(P, Q)+\psi_{\mu \nu} J_{3}(P, Q)
$$

In the Appendix we will show, explicitly, how $J_{1}, J_{2}$ and $J_{3}$ can be calculated from (4.48). Explicit differentiation of the left-hand side of Eq. $(4.47)$ gives the following set of equations for the scaled form factors

$$
\begin{aligned}
& \left(2+P \cdot \frac{\partial}{\partial P}\right)\left(1+P \cdot \frac{\partial}{\partial P}\right) A_{S}(P, Q)=J_{1}(P, Q) \\
& \left(1+P \frac{\partial}{\partial P}\right)\left(P \cdot \frac{\partial}{\partial P}\right) B_{S}(P, Q)=J_{2}(P, Q) \\
& \left(-1+P \cdot \frac{\partial P}{\partial P}\right)\left(P \cdot \frac{\partial}{O P}\right) \bar{C}_{S}(P, Q)=J_{3}(P, Q)
\end{aligned}
$$

The integration of $(4.50 \mathrm{~b})$ is straightforward 
48.

$B(P, Q)=B(Q, Q)+2 \int_{0}^{1} \frac{d \lambda_{1}}{\lambda_{1}} \int_{0}^{1} \frac{d \lambda_{2}}{\lambda_{2}} \int_{0}^{1} d \lambda_{3}\left[J_{2}\left(\lambda_{1} \lambda_{2} \lambda_{3} P_{1} \lambda_{1} Q\right)-J_{2}\left(Q_{1} \lambda_{1} Q\right)\right]$

$B(O, Q)$ can be determined from the Ward identity equation $(4.10)$

$$
B(Q) \equiv B(0, Q)=Q^{-2}\left[S^{\prime}(0)-\Delta^{\prime}(Q)\right]
$$

which, in tum, can be computed from Chapter II. By the same Ward identity, A(P, Q) is given in terms of $B(P, Q)$

$$
A(P, Q)=\frac{\left[\Delta^{-1}(P)-\Delta(P+Q)\right]}{2 Q \cdot e}-\frac{Q^{2}}{2 Q \cdot P} B\left(P_{1} Q\right)
$$

The integration of $(4.50 \mathrm{C})$ is also easy, and gives for $C(P, Q)$

$$
\bar{C}(P, Q)=2 \int_{0}^{1} \frac{d \lambda_{1}}{\lambda_{1}} \int_{0}^{1} \frac{d \lambda_{2}}{\lambda_{2}} \int_{0}^{1} \frac{d \lambda_{3}}{\lambda_{3}^{2}} J_{3}\left(\lambda_{1} \lambda_{2} \lambda_{3} P_{1} \lambda_{1} Q\right)
$$

Here we have used the fact that $C(O, Q)=0$ and that $\rfloor_{3}(O, Q)=0$ (for $(4.54)$ to be consistent, $J_{3}(O, Q)$ approaches 0 as $P^{2}$. This can be seen to be the case by inspecting (4.49)).

Equation (4.47), together with (4.5), (4.52), (4.53) and (4.54) [or in principle (4.39) and its integrated form ] represent an explicit solution to the first part of our program. 
49.

4.6 Determination of $C(O, Q)$

Now we will determine $C(O, Q)$ so that $Z(Q, P)+C(O, Q)=C(P, Q)$. As we hove already pointed out, we must study $(4.24)$ when $P=0$. As far as we were able to determine, the simplest differential operator which makes (4.24), with $P=0$, finite and removes the homogeneous term is

$$
\operatorname{Di}_{p}(Q)=\square_{p}^{2}=\frac{\partial}{\partial} \cdot \frac{\partial}{\partial \varphi}
$$

$C(O, Q)$ obeys then the differential-integral equation

$$
\begin{aligned}
& \square_{Q}^{2}\left[Q_{R,} C_{i}(Q, Q)\right]=\frac{1}{2}\left(\square_{Q}^{2} K_{S}\right)_{R=0}(\Delta \Delta)_{S} \bar{\theta}^{u v}+ \\
& +\frac{1}{2}(T)_{R=0}\left[\Delta_{Q}^{2}(\Delta \Delta)_{S}\right] \bar{\theta}^{n v}+\frac{1}{2}(T)_{R=0}(\Delta \Delta)_{S}\left(\nabla_{Q}^{2} K_{S}\right)\left(\Delta \Delta_{S} \bar{\theta}^{n V}\right.
\end{aligned}
$$

Equations (4.56) and (4.47) (or (4.39)) are a set of coupled integral differential equations with two independent unknown functions $A(P, Q)$ (and/or $B$ ) and $C(P, Q)$, which can be solved by iteration. Our separation of $\bar{\sigma}^{\mu N}$ into $\bar{\phi}^{\mu N}$ and its $P=0$ piece is then justified, for only one equation would not have solved the problem. The integration of (4.56) is also straightforward. Call the calculable, finite integral! in the right-hand side of (4.56) $H_{\mu N}(Q)$, so

$$
\square_{Q}^{2}\left[Q_{\mu \nu} C(Q, Q)\right]=H_{\mu \nu}(Q)=Q_{\mu \nu} h\left(Q^{2}\right)^{\prime}
$$

since $H_{\mu}$ depends only on the four-vector $Q$ : Doing the derivative in the left-hand 
side of $(4.57)$ yields the following differential equation for $C\left(Q^{2}\right)$ in terms of $k\left(Q^{2}\right)$

$$
\left(2+Q^{2} \frac{\partial}{Q^{2}}\right) Q^{2} \frac{\partial}{\partial Q^{2}} C\left(Q^{2}\right)=\frac{Q^{2} h\left(Q^{2}\right)}{4}
$$

This equation has the solution

$$
C(O, Q)=C_{0}+1 / 4 \int_{0}^{1} d y \int_{0}^{1} d x x^{2} h\left(x y Q^{2}\right)
$$

where we have used the fact that

$$
Q \frac{\partial}{\partial Q} G(Q Q)=0 \quad \therefore \text { AT } Q^{2}=0
$$

Otherwise, a singularity of $C\left(Q^{2}\right)$ at $Q^{2}=0$ would not be consistent with our results in the forward direction and the onalytic proporties of the propagator. Wo have also set

$$
\left.G(Q Q)\right|_{Q=0}=C_{0}
$$

where $c_{0}$ is an abitrary constant. Nowhere th our renomalization progrant hiove we found that $c_{0}$ should be equal to some well-defined number, like $V / 6$. This contradicts the CCJ result and some recent speculations in the literature (28). Furthermore, using (4.11) and (4. 12), we realize that no specific value of of will make the trace of $Q^{p N}$ soft. This shouldn't be surprising. The renormal ization processes are underlined by some sort of regularizotion procedures which introduce large mass torms into the theory. In the case of QED, these terms will not disturb gauge invarience, say, 
but for theories like the one under study, they will badly break its scale and conformal symmetries, which is reflected in the "non-softness" of the trace. The trace will become soft only when the strong requirement that there should be a solution to a GellMann-Low type eigenvalue equation for the bare coupling constant, is satisfied ${ }^{(26)}$. As we will show in a subsequent poper, the existence of an eigenvalue makes possible the construction of a conformal invariant theory. Within this context, the energymomentum tensor will be exactly the $C C J$ tensor with $c_{0}=1 / 6$, with its trace equal to zero, as it should. It is a general characteristic of conformal theories at the eigenvalue that they most resemble the "classical" theories without quantum corrections.

Let us finally remark that CCJ's failure in the renormalization proof came about because they made the critical assumption that the trace of the tensor was to be soft to all orders, which as we see is unjustified. Along their line of thought our proof is consistent since our renomalization was done with the tensor which has the softest trace of them all, namely the one with trace exactly equal to zero:

The problem of the energy-momentum tensor is to be considered clearly and explicitly solved. .

\section{Acknowledgments}

I would like to thank Professor. Kenneth A. Johnson who suggested many ideas in this paper and discussed them in a very illuminating manner.

The Theory Division of CERN and the M. I. T. Center for Theoretical Physics, where parts of this work were carried out, are gratefully acknowledged for their hospitality. 


\section{Appendix}

Explicit Determination of the Traceless Energy-Momentum Tensor $-\mu N$ Form-Factors

We want to calculate the finite functions $J_{1}(P, Q), J_{2}(P, Q)$ and $J_{3}(P, Q)$

which are necessary to calculate $A(P, Q), B(P, Q)$ and $C(P, Q)$ from $E_{q i}$ (4.49).

$J_{1}(P, Q), J_{2}(P, Q)$ and $J_{3}(P, Q)$ are defined by the integral

$J_{\mu v}\left(P_{i} Q\right) \equiv P_{\mu v} J_{1}\left(P_{1} Q\right)+\left(P_{Q}\right)_{\mu v} J_{2}(P, Q)+\left(Q_{M v}\right) J_{3}\left(P_{1} Q\right)=$

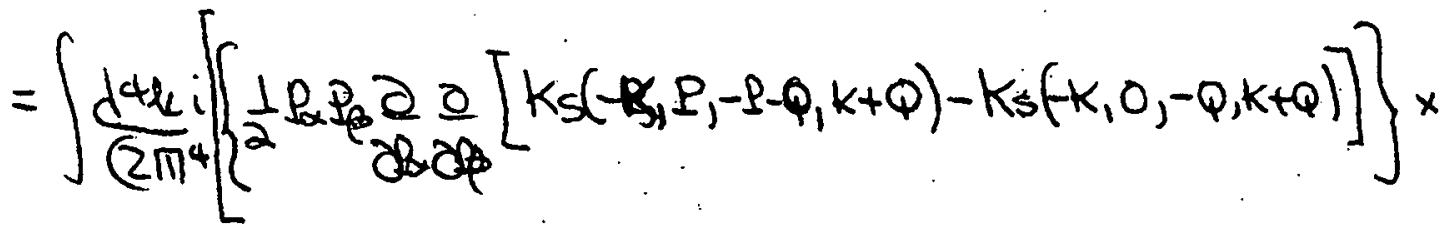

$\left.\times\left\{\Delta(k) \bar{\theta}^{\omega \prime}(k, k+\varphi) \Delta(k+\varphi)\right\}\right]$

The spectral decomposition of the integrand with respect to the direction $\hat{k}$, will depend on the projections along $\hat{P}, \hat{Q}$ and some arbitrary direction (introduced by the tensor nature of the integrand). The angular integration with respect to $\hat{k}$ will, then, distinguish three well-defined integrals which are linear combinations of $j_{1}$, $J_{2}$ and $J_{3}$ which can thus be determined by solving the resulting set of oquations. Specifically, define the following three distinct integrals

$$
\begin{aligned}
& I_{1}(P, Q)=\frac{\partial P_{\mu} P_{v}}{(1-x) P^{4}} J^{x^{v}}(P, Q) \\
& I_{0}(P, Q)=\frac{\left(P_{\mu} Q v+P_{\mu} Q_{\mu}\right)}{P^{2} Q^{2}(1-x)} J^{\mu \nu}(P, Q)
\end{aligned}
$$


53.

$I_{3}(P, Q)=\frac{2 Q, Q v}{(1-X) Q^{4}} J^{W^{J}}(P, Q)$

(A. Ic)

where

$$
X=\frac{(P \cdot Q)^{2}}{P^{2} Q^{2}}
$$

... then, using (A. I) and solving the system of equations we get

$$
\begin{aligned}
& J_{1}(P Q)=\frac{3}{4(1-x)}\left\{I_{1}-\frac{2 P Q}{P^{2}} I_{2}+\frac{(1+2 x)}{3} \frac{Q^{2}}{P^{2}} I_{3}\right\} \\
& J_{2}(P, Q)=\frac{3}{2(1-x)}\left\{-\frac{P Q}{Q^{2}} I_{1}+\frac{2}{3}(1+2 x) I_{2}-\frac{P Q}{P^{2}} I_{3}\right\} \\
& J_{3}(P, Q)=\frac{3}{4(1-x)}\left\{\frac{(1+2 x)}{3} \frac{P^{2}}{Q^{2}} I_{1}-\frac{2 P Q}{Q^{2}} I_{2}+I_{3}\right\}
\end{aligned}
$$

The integrals $I_{1}, L_{2}, I_{3}$ can be done from (A. 1) by using the properties (3,28), (4.35) and (4.36) of the angular functions $C_{n}(z)$. 


\section{References}

1. Wu, T. T.: : Phys. Rev. 125, 1436 (1962)

2. Bogoliubov, N.N.; Shirkov, D.V.: Introduction to the Theory of Quantized Fields... Now York, Interscience Publ. (1957).

3. Mills, R. L.; Yang, C. N. : Progr. Theor. Phys. (Kyoto) Supp. 37-38 (1966)

4. Taylor, 1.G.: Suppl. al Niovo Cimento 1, 857 (1963)

5. Johnson, R.W.: J. Math. Phys. 11, 2161 (1970)

6. Zimmermann, W. : Lectures on Elementary Perticles and Quantum Fidd Thoory. V. I. Ed. by Deser, Grisoru and Pendloton. M. I. T. Pross (1971)

7. Symanzik, K. : Comm. Math. Phys. 18, 227 (1970)

8. Callan Jr., C. C. : Phys. Rey. D2, 1541 (1970)

9. Wilson, K.: Phys. Rov. D2, 1473 (1970)

10. Poggio, E. C.: M. L.T. Ph. D. Thesis, September 1971.

11. Barut, A. O.; Mullen, G. H.: Ann. Phys. (N. Y.) 20, 203 (1962)

12. Nordstrom, D.L., Phys. Rev. D4, 1611 (1971)

13. Gell-Mánn, M.; Low, F.: Phys. Rev. 95, 1300 (1954)

14. Wilson, K.: Phys. Rev. D8, 1818 (1971)

15. Baker, M.; Johnson, K.: Phys. Rev. 183, 1292 (1969)

16. Adler, S.: Phys. Rev. D5, 3021 (1972)

17. Johnson, K.: Proceedings of Lectures Commemorative to W. Hoisenberg, Ed. by H. Durr (1972)

18. Callan Jr., C. C.; Coleman, S.; Jackiw, R. : Ann. of Phys. (N. Y.) 59, 42 (1970)

19. Poggio, E. : to be published 
20. Blankenbeckler, R.: 1968 Rochester Conference Proceedings, Ed, by Guralnik and Hagen.

21. Nakanishi, N.: Prog. Theor. Phys. (Kyoto) 25, 155 (1960)

22. Ward, J. C. : Phys. Rev. 84,897 (1954)

23. Boym, G.: Phys. Lett. I, 241 (1962)

24. Coleman, S.; Jackiw, R. : Ann. Phys. (N. Y.) 67, 552 (1971)

25. Lowenstein, J. : Phys. Rev. O4, 2281 (1971)

26. Schroer, B. : Lett. al Nuovo Cimento 17, 867 (1971)

27. Zumino, B. : Lecturos in Elementary Particles and Quantum Fiold Thoory, V. 2.

Ed. by Deser, Grisaru and Pendleton. M. I.T. Press (1971)

28. Milton, K. A.: Phys. Rev. D4, 3579 (1971) 


\section{Figure Captions}

Figure 1 The propagator and the vertex function

Figure 2 Examples of O.P. I. and O.P.R. propagator and vertex graphs

Figure 3 Examples of primitive and non-primitive graphs

Figure 4 Form of T.P.R. and T.P. I. vertex

Figure 5 The vertex function and its momentum space invariants

Figure 6 The V-graphs (T.P. L. vertex)

Figure 7 Graphical (formal) equation for the vertex

Figure 8 Lowest order graphical solution to $K_{S}$ and $T$

Figure 9 Equation for the differentiated vertex function

Figure $10^{\circ}$ Some lowest order contributions to the set of O.P.I. self-energy graphs

Figure 11 Integral equation for $\theta^{\mu N}(P, P)$

Figure 12 Integral equation for $\bar{\theta}^{N N}(P, P+Q)$ in terms of $K_{S}$

Figure 13 Integral equation for $\bar{\theta}^{\mu N}(P, P+Q)$ in terms of $T$

Figure 14 Integral equation for the differentiated $\bar{\theta}^{-N}(P, P+Q)$ without homogeneous-terms dependent terms 


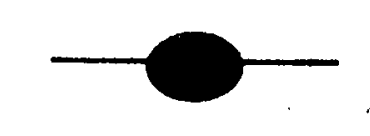

PROPAGATOR

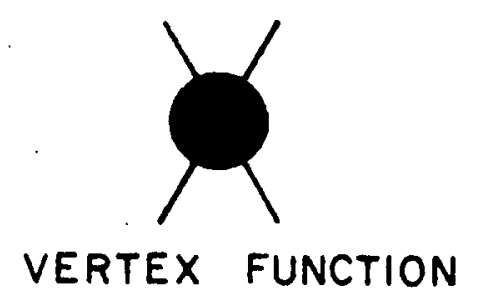

FIGURE 1 


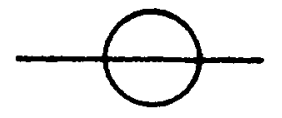

O.P.I PROPAGATOR

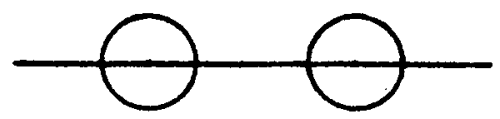

O.P.R PROPAGATOR

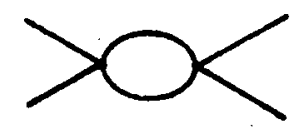

O.P.I VERTEX

FIGURE 2

1
$i$
2
0
2
8 


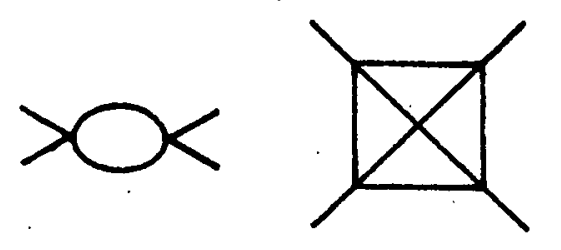

PRIMITIVE GRAPHS
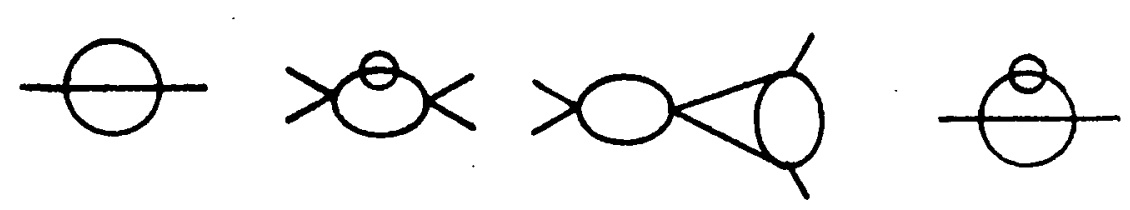

FIGURE 3 


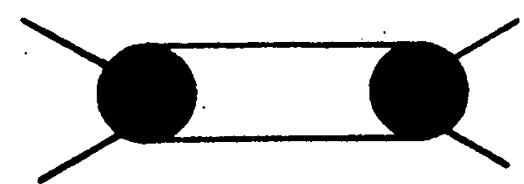

T.P.R. VERTEX

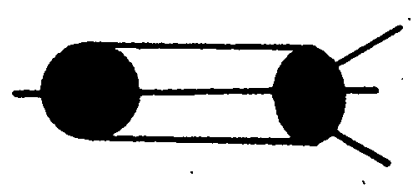

T.P. I. VERTEX

FIGURE 4 

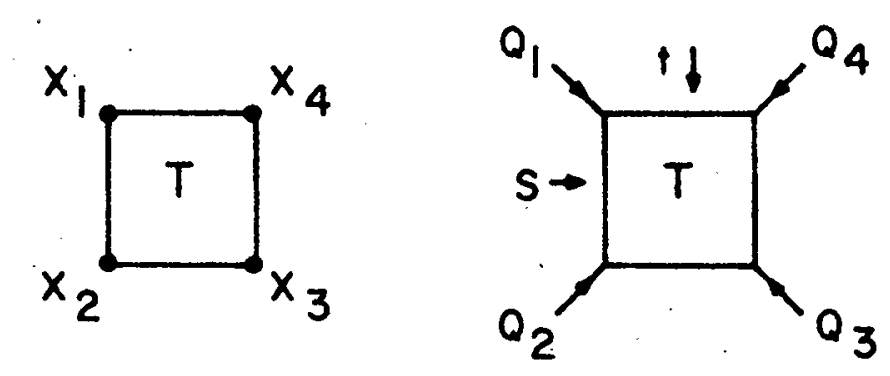

$\vdots$
$\vdots$
0
$\vdots$
$\vdots$
$\vdots$
$\vdots$

FIGURE 5 


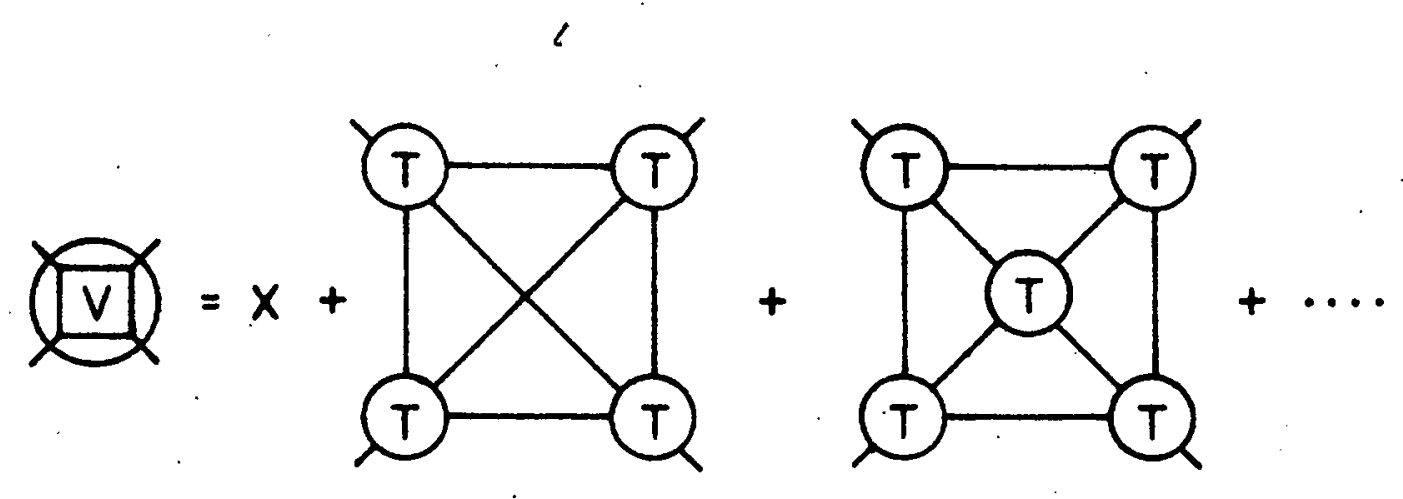

FIGURE 6 

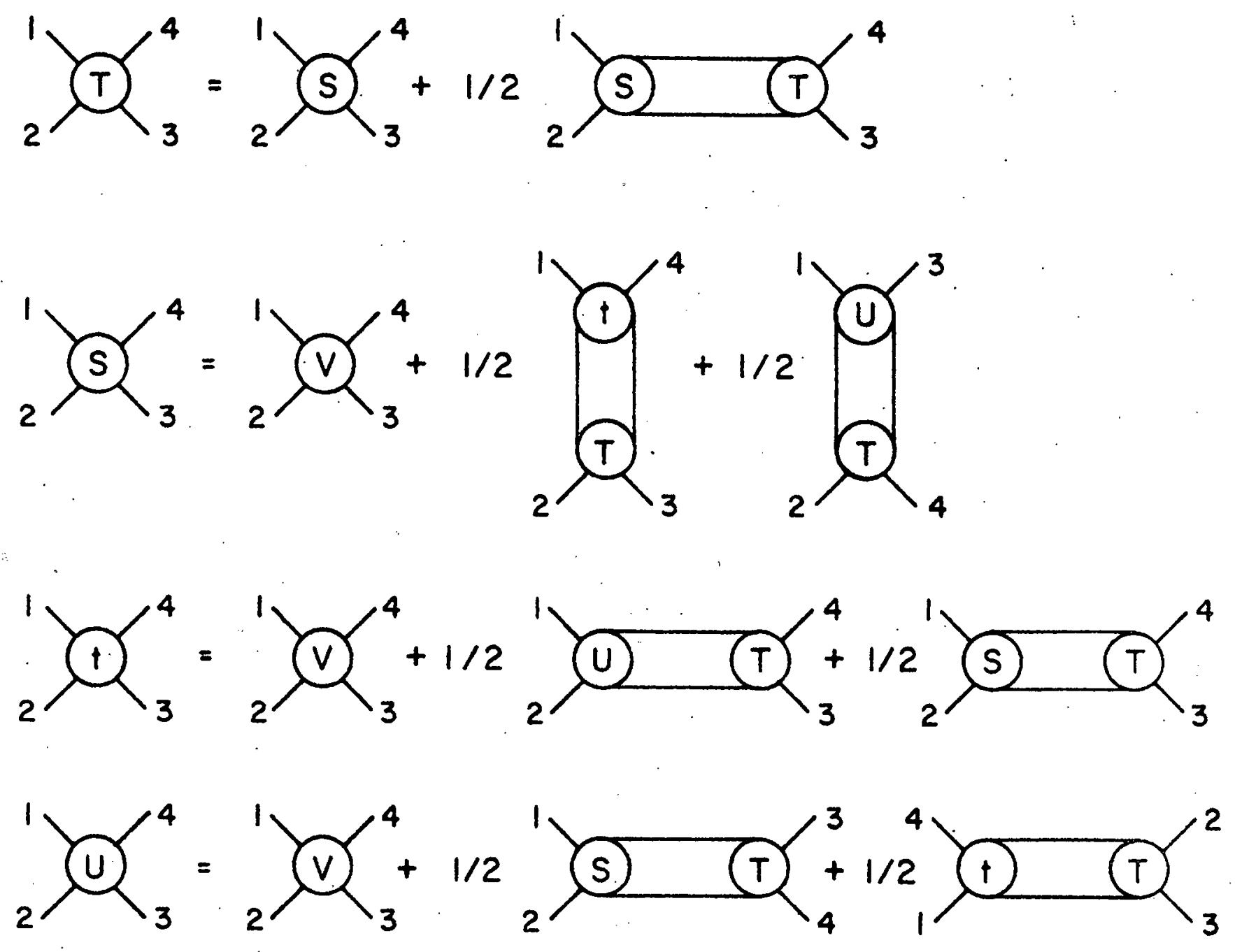


$$
\begin{aligned}
& { }_{2}^{1} S^{4} X_{3}^{4}={ }_{2}^{1} X_{3}^{4}+1 / 2{ }_{2}^{1} X_{3}^{4}+1 / 2{ }_{2}^{1} X_{4}^{3}+1 / 2{ }_{2}^{1} X_{3}^{4}+1 / 2{ }_{2}^{1} X_{3}^{4} \\
& +1 / 2{ }_{2}^{1} \chi_{4}^{3}+1 / 2{ }_{2}^{1} X_{4}^{3}+1 / 4 \gamma_{2}^{1} X_{3}^{4}+1 / 4 \gamma_{2}^{1} \gamma^{3}+\cdots
\end{aligned}
$$$$
{ }_{2}^{1} \gamma_{3}^{4}={ }_{2}^{1} X_{3}^{4} X_{3}^{+1 / 2}{ }_{2}^{1}>\alpha_{3}^{4}+{ }^{1}{ }^{1} X_{2}^{4} X_{3}+1 / 2{ }_{2}^{1} X^{3}+
$$$$
{ }_{+1 / 2}^{1}{ }_{2}^{1} \alpha_{3}^{4}+{ }_{2}^{1}{ }_{2}^{1} \gamma_{3}^{4}+{ }_{1}^{1}{ }_{2}^{1} \gamma_{4}^{3}+
$$$$
{ }_{+1 / 2}^{1}>Q_{3}^{4}+1 / 2{ }_{2}^{1} X_{3}^{4}{ }_{2}^{1 / 2} X^{3}{ }^{+}
$$$$
+1 / 4{ }_{2}^{1} \gamma_{3}^{4}{ }^{4}+1 / 4{ }_{2}^{1} X_{4}^{3} X^{3}+1 /{ }_{2}^{1}>00 \alpha_{3}^{4}+\cdots
$$

FIGURE 8 


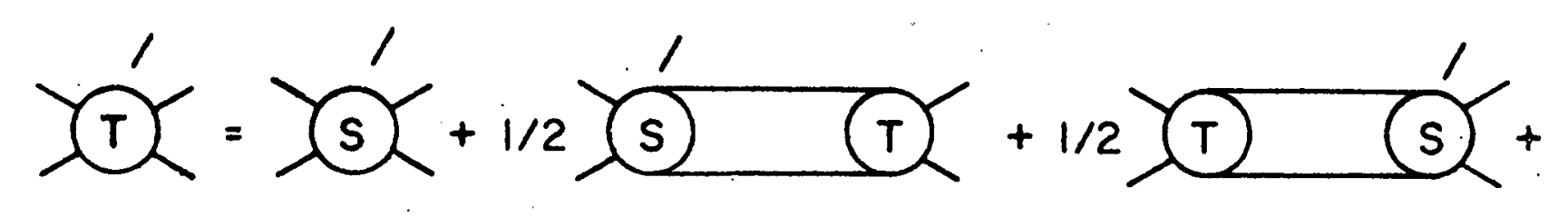

$+1 / 2)(T)$ (T) + 1/4 (T) (s) (T 



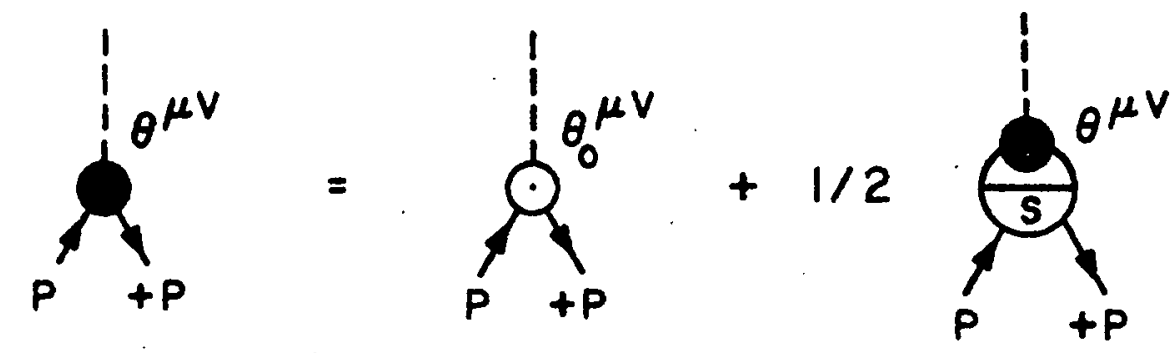

FIGURE 11 


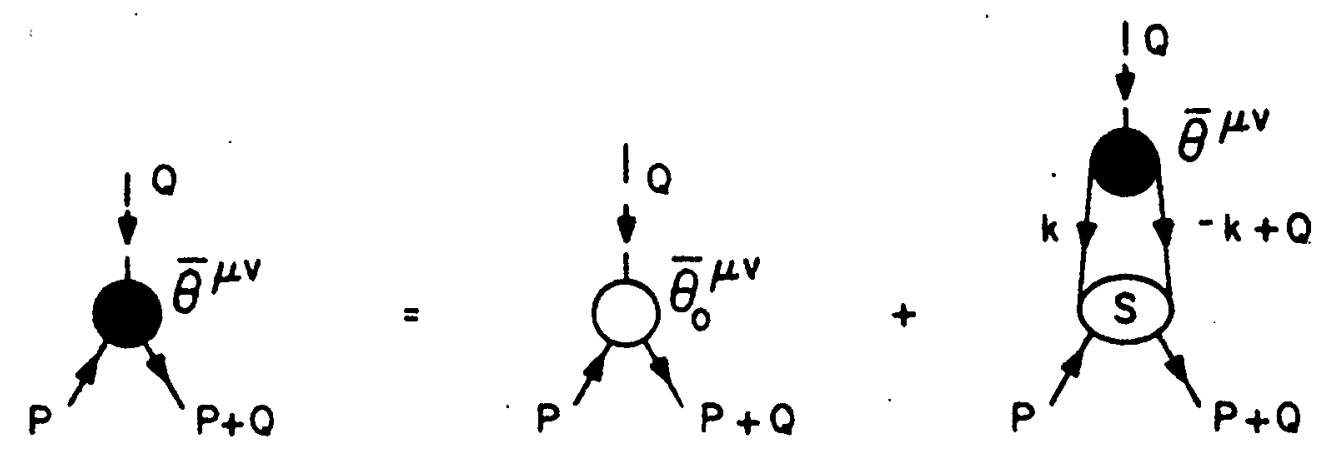

FIGURE 12 

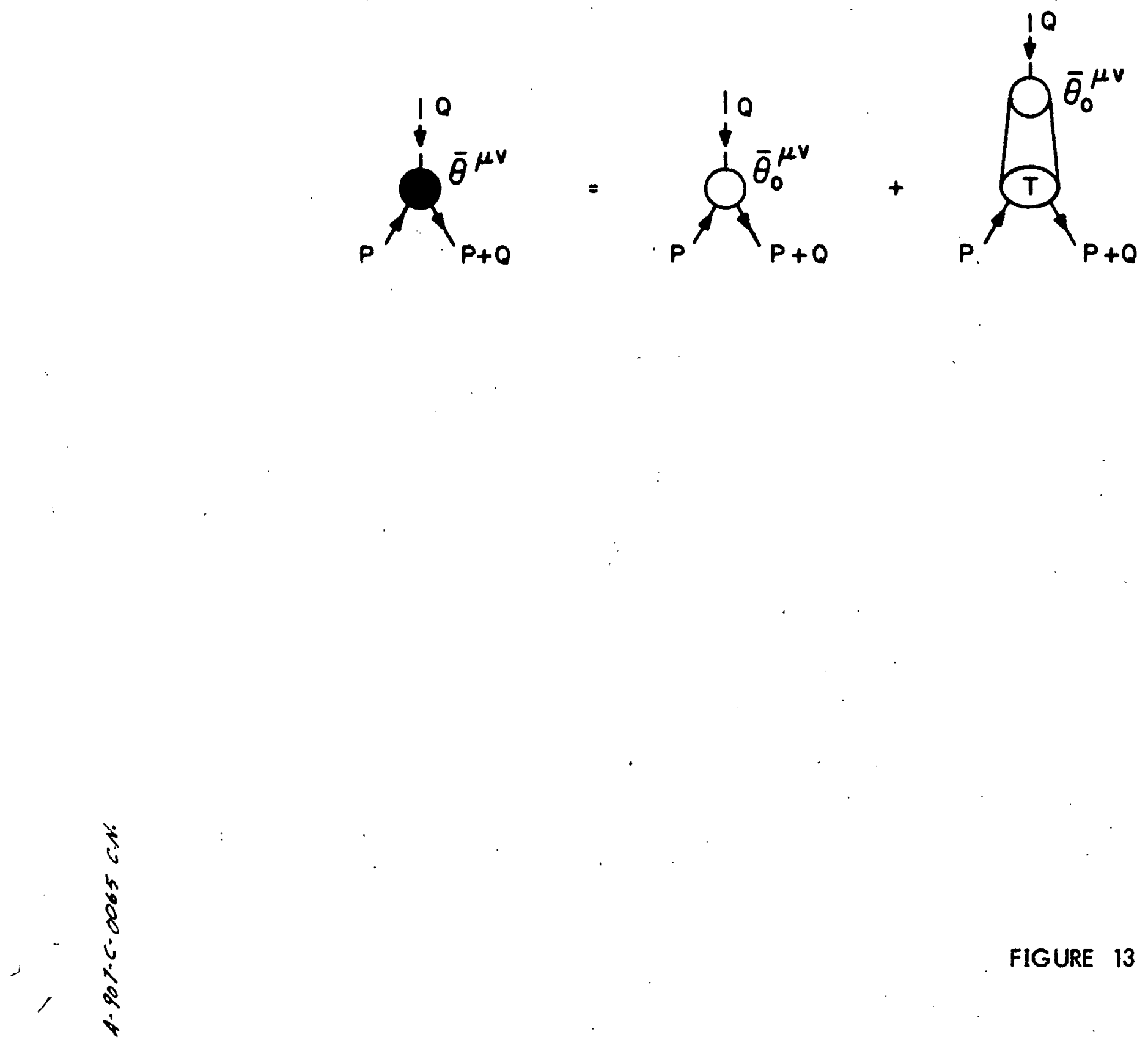

FIGURE 13 

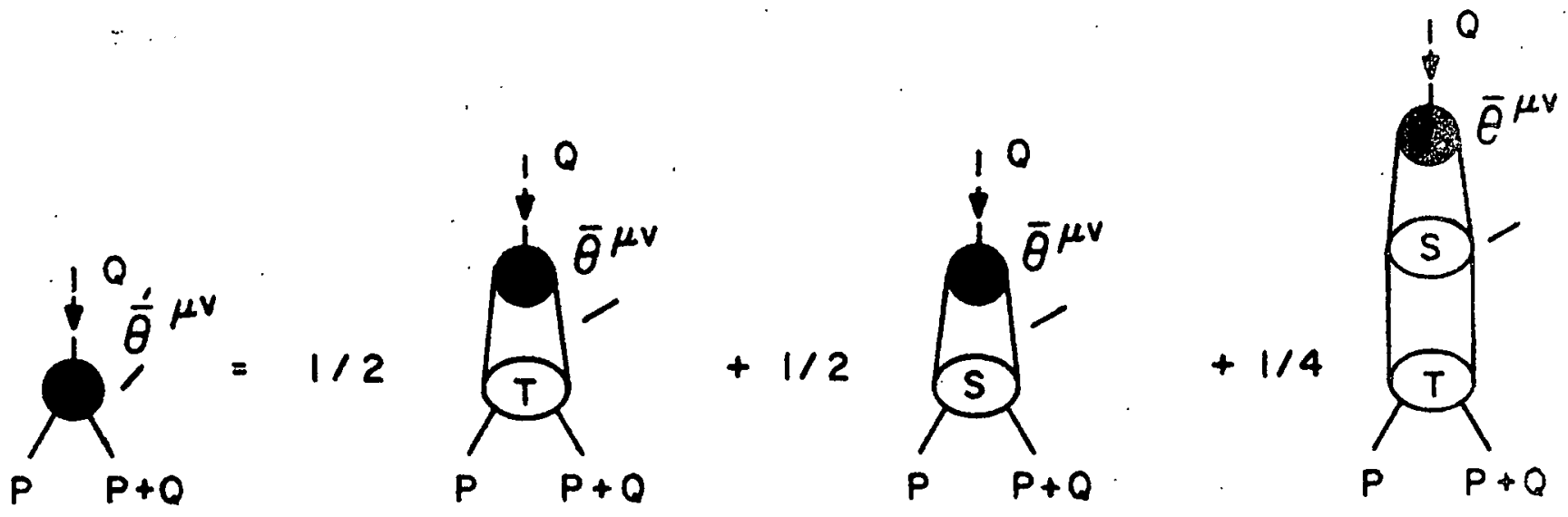